\title{
A catalytic and tert-butoxide ion-mediated amidation of aldehydes with para-nitro azides
}

\author{
Giorgio Carbone, James Burnley and John E. Moses*
}

${ }_{5}$ Published in Chem. Commun 2013, 49, 2759

\section{DOI: $10.1039 / \mathrm{b} 000000 \mathrm{x}$}

We report here a new catalytic reaction in which, $p$-nitro azides are acylated by aldehydes to produce amides and molecular nitrogen in a single step. The transformation is believed to proceed via an electron transfer process mediated by the tert-butoxide ion, and catalysed by a thiazolium salt derived species.

10 Amide bond forming reactions are among the most executed in organic chemistry, forming key linkages in peptides, proteins, synthetic polymers and drugs. ${ }^{1}$ Traditional approaches to amide synthesis involving the coupling of activated carboxylic acid derivatives (anhydrides and acyl chlorides) with nucleophilic amines are expensive, wasteful and often produce toxic by-products necessitating lengthy purification. ${ }^{2}$ The demand for sustainable and greener ${ }^{3}$ approaches to amide synthesis has stimulated intense activity in the development of new and creative catalytic methods. ${ }^{1 \mathrm{~d}}$

15 Straightforward and desirable methods involving the direct coupling of carboxylic acids and amines with certain boronic acid catalysts have been reported. ${ }^{4}$ Other strategies generally involve either the catalytic or oxidative acylation of an amine, or occur by a suitable combination of complementary reaction partners following a unique pathway. For example, the catalytic generation of activated carboxylates from functionalised aldehydes by $\mathrm{N}$-heterocyclic carbene (NHC) catalysts with a co-catalyst, followed by their conversion to amides has been shown to work with a variety of amines. ${ }^{5}$ Oxidative processes utilising $\mathrm{NHC}^{\prime} \mathrm{s}^{6}$ and metal based catalysts have been 20 used for amide formation from aldehydes with stoichiometric oxidants. ${ }^{7}$ The ruthenium catalysed conversion of alcohols and primary amines with loss of $\mathrm{H}_{2}$ developed by Milstein et al. ${ }^{8}$ represents an example of an atom economic and green approach to amide synthesis, whereas the oxidative coupling of $\alpha$-bromo nitroalkanes with amines offers a quite different pathway. ${ }^{9}$

The formation of amides by the intermolecular coupling of thioacids and electron poor anilines that require no activating or coupling reagents has been investigated. ${ }^{10,11}$ The mechanism is believed to involve the nucleophilic attack of the thioacetate onto the azide $N-3$

${ }_{25}$ followed by formation of a cyclic thiatriazoline intermediate which collapses to give the amide, nitrogen gas and elemental sulfur ${ }^{.11}$ In seeking to further develop the theme of this chemistry, we were intrigued by the possibility of using aldehydes directly in a redox azido-amidation type-process, thus broadening the range of available substrates and eliminating the sulfur by-product.

In recent studies, we observed that in the presence of the thiazolium salt (2), the tert-butoxide ion selectively reduces the azide group of para-azidonitrobenzene (Scheme 1 ). ${ }^{12}$ The reaction is believed to proceed by an electron transfer process via the dianion $\mathbf{3}$, followed 30 by concomitant loss of nitrogen gas to give aniline 4 .

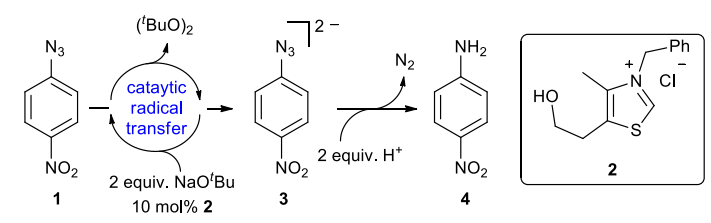

Scheme 1 A proposed mechanism to explain the catalytic reduction of 1 to aniline 4 by the -butoxide ion.

${ }_{35}$ We now report the tert-butoxide ion mediated amidation of aldehydes ${ }^{13}$ with $p$-nitro azides, catalysed by a thiazolium salt derived catalyst. This clean transformation allows the synthesis of 4-nitroaromatic amides in one step with high atom economy, and driven by loss of environmentally benign nitrogen gas. ${ }^{14}$

For the reaction development we opted to use the electron deficient 1-azido-4-nitrobenzene 1, with benzaldehyde 5 as the acyl donor. Using previously optimised conditions for the azide-reduction as a starting point ( 2 equiv. tert-BuONa, THF, rt), ${ }^{12}$ a number of 40 preliminary reactions were performed (Table 1 ).

Thus, treatment of the azide $\mathbf{1}$ and aldehyde $\mathbf{5}$ in THF with tert-BuONa at room temperature immediately resulted in the evolution of a gas and, complete conversion of the starting material 1. After chromatography, the corresponding target amide 6 was isolated in $56 \%$ yield along with $p$-nitroaniline 4 (39\%) (Table 1, Entry 1). Similar results were achieved when the potassium salt of tert-butoxide ion was employed; giving amide 6 (51\%) and aniline 4 (36\%) (Table 1, Entry 2). However, when the same reaction was carried out in the 
presence of 1 equiv. of the thiazolium salt 2, the yield of the amide 6 decreased (30\%) whereas the aniline 4 was isolated in higher yield (62\%) (Table 1, Entry 3).

When a solution of $\mathbf{1}$ and $\mathbf{5}$ was treated with tert-BuONa ( 2 equiv.) at $-25{ }^{\circ} \mathrm{C}$ without catalyst, unreacted starting materials were recovered (Table 1, Entry 4). In contrast, the addition of $\mathbf{2}$ ( 1 equiv.) to the reaction mixture at $-25^{\circ} \mathrm{C}$ followed by tert-BuONa ( 3 equiv.) ${ }_{5}$ gave the target amide 6 in $80 \%$ yield with no observed aniline 4 (Table 1, Entry 5). A reduced loading of 2 (0.1 equiv.) resulted in a similar yield of amide 6 (81\%) (Table 1, Entry 6).

Stirring a mixture ( $30 \mathrm{~min}$ ) of the azide 1 , the aldehyde 5 and tert-BuONa ( 2 equiv.) at $-25{ }^{\circ} \mathrm{C}$; no reaction was observed (TLC) until the addition of $\mathbf{2}$ ( 0.1 equiv.), then after a further $30 \mathrm{~min}$, the amide 6 was isolated in $83 \%$ yield (Table 1, Entry 7).

Table 1 Optimisation of the reaction conditions and control experiments.

\begin{tabular}{cccccc}
$1(0.5 \mathrm{mmol})$ \\
\hline Entry 2 (equiv.) base (equiv.) $\mathrm{T}\left({ }^{\circ} \mathrm{C}\right)$ & $\mathbf{6}$ yield $^{a}(\%)$ & $\mathbf{4}$ yield ${ }^{a}(\%)$ \\
\hline 1 & - & $\mathrm{NaO}{ }^{t} \mathrm{Bu}(2.0)$ & $\mathrm{rt}$ & 56 & 39 \\
2 & - & $\mathrm{KO}^{t} \mathrm{Bu}(2.0)$ & $\mathrm{rt}$ & 51 & 36 \\
3 & 1.0 & $\mathrm{NaO}{ }^{t} \mathrm{Bu}(3.0)$ & $\mathrm{rt}$ & 30 & 62 \\
4 & - & $\mathrm{NaO}{ }^{t} \mathrm{Bu}(2.0)$ & -25 & 0 & 0 \\
5 & 1.0 & $\mathrm{NaO}{ }^{t} \mathrm{Bu}(3.0)$ & -25 & 80 & 0 \\
6 & 0.1 & $\mathrm{NaO}{ }^{t} \mathrm{Bu}(2.0)$ & -25 & 81 & 0 \\
7 & $0.1^{b}$ & $\mathrm{NaO}{ }^{t} \mathrm{Bu}(2.0)$ & -25 & 83 & 0 \\
\hline
\end{tabular}

${ }^{a}$ isolated yield after chromatography; ${ }^{b} \mathbf{2}$ added after stirring $\mathbf{1}$ and $\mathbf{2}$ at $-25^{\circ} \mathrm{C}$ in the presence of tert-BuONa.

We next investigated the one pot diazotisation-azidation-amidation reaction, since this would negate the isolation of the azide substrate. ${ }^{15}$ This proved viable; the diazotisation/azidation of $p$-nitroaniline 4 with ${ }^{t} \mathrm{BuONO} / \mathrm{TMSN}_{3}$ at $0{ }^{\circ} \mathrm{C}$ in $\mathrm{THF}^{16}$ followed by catalytic amidation at $-25{ }^{\circ} \mathrm{C}$ provided the amide 6 in an excellent $94 \%$ yield (Table 1, Entry 1 ). The scope of the tandem reaction was next 15 investigated with a number of electron deficient anilines and several aldehydes (Table 2, Entries 1-15). Both ortho- and metasubstituents on the para-nitroaromatic azide were well tolerated with benzaldehyde (Table 2, Entries 2-4). The reaction worked best when electron deficient anilines were coupled with electron rich aromatic aldehydes, including ortho- and para-substitution on the aromatic aldehyde (Table 2, Entries 5-7).

When an electron deficient aromatic aldehyde was used, the yield of amide dropped significantly (38\%) (Table 2, Entry 8). The 20 heteroaromatic thiophene-2-carbaldehyde was tolerated, giving the corresponding amide isolated in good yield (82\%) (Table 2, Entry 9). When alkyl aldehydes were employed; high yields were consistently obtained (78-94\% yield, entries 10-15).

The reaction raises a number of interesting questions with regard to the mechanism. During the 1980's, Guthrie and co-workers published a number of detailed papers on the reaction mechanism between the tert-butoxide ion and nitrobenzene, involving SET processes. ${ }^{17}$ Indeed, the function of tert-butoxide ion as an electron transfer agent is well documented. ${ }^{18}$

${ }_{25}$ When the amidation reaction of the azide $\mathbf{1}$ and benzaldehyde (2) was attempted under an atmosphere of oxygen or in the presence of the radical trap TEMPO, no reaction was observed with complete recovery of the azide 1 . These results, which are consistent with our earlier findings ${ }^{12}$ provide support for a radical based mechanism in the amidation process (See Scheme S1, supporting information (SI)).

Table 2 Reaction of para-nitro aromatic azides with various aldehydes

Aniline Aldehyde


4
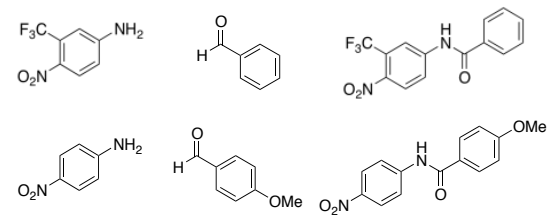<smiles>Nc1ccc([N+](=O)[O-])cc1</smiles><smiles>COc1ccc(C(=O)Nc2ccc([N+](=O)[O-])cc2)c(OC)c1</smiles>
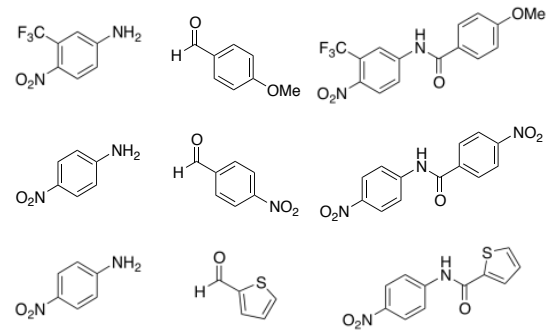

10
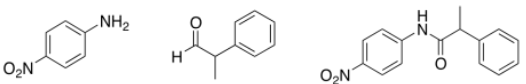

11
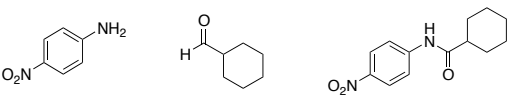

12

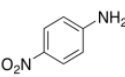<smiles>O=CC1CC1</smiles>

13
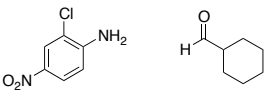

14

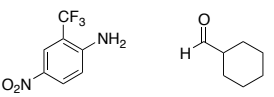

15<smiles>O=C(Nc1ccc([N+](=O)[O-])cc1)C1CC1</smiles>

${ }^{a}$ isolated yield after chromatography.

The relative nitrogen connectivity between the starting azide and the corresponding amide was determined by performing experiments with ${ }^{15} \mathrm{~N}$-labelled 1-azido-4-nitrobenzene. The reaction proceeded with retention of ${ }^{15} \mathrm{~N}$ directly attached to the aromatic ring (See Scheme S2, SI).

${ }_{5}$ The role of the thiazolium salt $\mathbf{2}$ is uncertain and warrants further investigation. However, preliminary mechanistic studies with the thiazolium derived triazene $7^{12,20}$ demonstrated that such speices are stable to the amidation conditions and hence ruled out as a possible intermediate (Scheme 2).

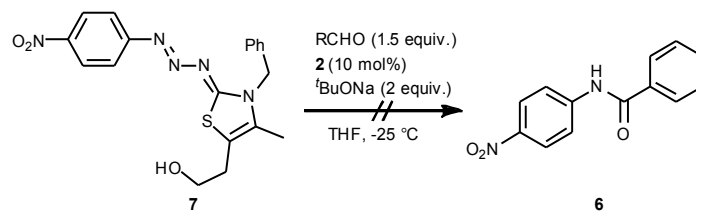

Scheme 2 Subjecting the triazene 7 to the preferred amidation conditions did not lead to the formation of $\mathbf{6}$. 


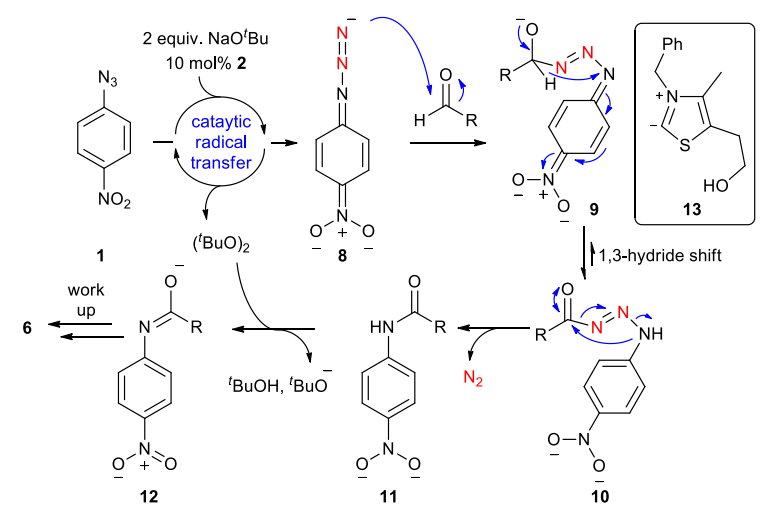

Scheme 3 A plausible amidation mechanism $1(\mathrm{R}=\mathrm{Ph})$.

Based upon the available experimental data, we present a working hypothesis to explain the mechanism. Thus, electron transfer from the tert-butoxide ion gives the dianion $\mathbf{8}$, a process that we tentatively propose is catalysed by a thiazolium anion relay derived from $\mathbf{2}$ ${ }_{5}(\mathbf{1 3}) .{ }^{12}$ Whereas protonation and loss of nitrogen from 8 would deliver the corresponding aniline (Scheme 1), interception of the intermediate 8 by an electrophilic aldehyde would deliver intermediate $9 .{ }^{14}$ A subsequent 1,3-hydride shift leads to intermediate 10 that is poised to extrude nitrogen gas and deliver the dianion 11. Next, in a manner consistent with the observations of Guthrie, ${ }^{17}$ we speculate that the loss of two electrons from 11 occurs through two SET processes with concomitant formation of two equivalents of the tert-butoxide anion from tert-butylperoxide. Overall imidate $\mathbf{1 2}$, tert-butanol and tert-butoxide are generated which, upon protic 10 work-up delivers the corresponding amide 6 and tert-butanol (Scheme 3 ).

\section{Conclusions}

In summary, we have documented a new and straightforward synthetic method for the catalytic synthesis of substituted $p$ nitroaromatic amides, based upon an azido-amidation mechanism. This method offers an orthogonal approach to current methodology and, in particular, the method works particularly well with electron deficient anilines, thus complementing existing methodologies 15 involving activated carboxylic acid derivatives. Studies are currently ongoing to unravel the mechanistic details of the reaction.

\section{Notes and references}

${ }^{a}$ School of Chemistry, University of Nottingham, England, UK, NG7 2RD; Te;: +44 (0)115951 3533; E-mail: john.moses@nottingham.ac.uk

+ Electronic Supplementary Information (ESI) available: Full experimental procedures and characterisation with ${ }^{1} \mathrm{H}$ and ${ }^{13} \mathrm{C}$ NMRs and schemes referenced in the text.. See DOI: 10.1039/b000000x/

20

1 (a) R. C. Larock, Comprehensive Organic Transformations, Wiley-VCH, New York, 2nd edn., 2010; (b) A. Greenberg, C. M. Breneman and J. F. Liebman, The Amide Linkage: Structural Significance in Chemistry, Biochemistry, and Materials Science, Wiley-interscience, 2002; (c) S. D. Roughley and A. M. Jordan, J. Med. Chem., 2011, 54, 3451-3479; (d) V. R. Pattabiraman and J. W. Bode, Nature, 2011, 480, $471-479$.

2 M. B. Smith, Compendium of Organic Synthetic Methods, Wiley, New York, Vol 12, 2009, 100-116.

253 D. J. C. Constable, P. J. Dunn, J. D. Hayler, G. R. Humphrey, J. L. Leazer, R. J. Linderman, K. Lorenz, J. Manley, B. A. Pearlman, A. Wells, A. Zaks and T. Y. Zhang, Green Chem., 2007, 9, 411-420.

4 (a) K. Ishihara, S. Ohara and H. Yamamoto, J. Org. Chem., 1996, 61, 4196-4197; (b) K. Ishihara, Tetrahedron, 2009, 65, 1085-1109; (c) T. Marcelli, Angew. Chem. Int. Ed., 2010, 49, 6840-6843; (d) P. Tang, Org. Synth., 2005, 81, 262-268; (e) H. Charville, D. Jackson, G. Hodges and A. Whiting, Chem. Commun., 2010, 46, 1813-1823.

305 (a) J. W. Bode and S. S. Sohn, J. Am. Chem. Soc., 2007, 129, 13798- 13799; (b) P.-C. Chiang, Y. Kim and J. W. Bode, Chem. Commun., 2009, 4566-4568; (c) H. U. Vora and T. Rovis, J. Am. Chem. Soc., 2007, 129, 13796-13797.

6 S. D. Sarkar and A. Studer, Org. Lett., 2010, 12, 1992-1995.

7 (a) Y.Tamaru, Y. Yamada and Z. Yoshida, Synthesis, 1983, 6, 474-476; (b) C. L. Allen and J. M. J. Williams, Chem. Soc. Rev., 2011, 40, 3405-3415;

(c) K. Ekoue-Kovi and C. Wolf, Chem. Eur. J., 2008, 14, 6302-6315.

358 C. Gunanathan, Y. Ben-David and D. Milstein, Science, $2007,317,790-792$.

9 B. Shen, D. M. Makley and J. N. Johnston, Nature, 2010, 465, 1027-1032.

10 (a) P. Sharma, A. D. Moorhouse and J. E. Moses, Synlett, 2011, 16, 2384-2386; (b) C. Spiteri and J. E. Moses, Synlett, $2012,23,1546-1548$.

11 (a) T. Rosen, I. M. Lico and D. T. W. Chu, J. Org. Chem., 1988, 53,

1580-1582; (b) N. Shangguan, S. Katukojvala, R. Greenberg and L.

40 J. Williams, J. Am. Chem. Soc., 2003, 125, 7754-7755.

12 J. Burnley, G. Carbone and J. E. Moses, unpublished results.

13 H. Inoue and K. Higashiura, J. C. S. Chem. Commun., 1980, 549550.

14 During the preparation of this manuscript, Manetsch et al., reported a novel, complementary tert-butoxide ion mediated amidation of

$45 \quad$ aldehydes with benzylic azides: S. S. Kulkarni, X. Hu and R. Manetsch, Chem. Commun., 2013, 49, 1193-1195. 
15 S. Bräse and K. Banert, Organic Azides: Syntheses and Applications, John Wiley \& Sons, inc., 2009.

16 K. Barral, A. D. Moorhouse and J. E. Moses, Org. Lett., 2007, 9, 1809-1811.

17 (a) R. D. Guthrie and D. L. Nutter, Bull. Soc. Chim. Belg., 1982,

91, 455; (b) R. D. Guthrie and D. L. Nutter, J. Am. Chem. Soc., 1982, 104, 7478-7482. (c)

18. A Russel, E. G. Jenzen and E. T Strom, J. Am. Chem. Soc., 86, 1964, 1807-1814.

19 (a) C. Tang and N. Jiao, J. Am. Chem. Soc., 2012, 134, 18924-18297;

(b) D. Qiu, L. Jin, Z. Zheng, H. Meng, F. Mo, X. Wang and Y.

Zhang, J. Org. Chem., 2013, DOI: 10.1021/jo3018878.

1020 (a) D. M. Khramov and C. W. Bielawski, Chem. Commun., 2005, 4958-4960; (b) D. M. Khramov and C. W. Bielawski, J. Org. Chem., 2007, 72, 9407-9417.

tert-Butoxide Promoted One-Pot Azidation-Amindation of paranitroanilines with aldehydes

${ }_{20}$ Giorgio Carbone, James Burnley and John E. Moses*

School of Chemistry, University of Nottingham, Nottingham, England,

UK, NG7 2RD

John.moses@nottingham.ac.uk Towhom correspondence should be addressed.

Schemes and Figures

Materials and Methods

30

Experimental Procedures

References

${ }_{35}$ NMR Spectra
S2

S3

S4-S12

S13

S14-29 


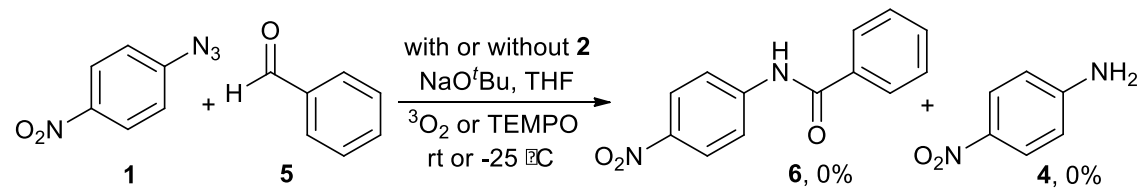

Scheme S1 Evidence for an electron transfer process in the azido-amidation reaction of azide $\mathbf{1}$ as both ${ }^{3} \mathrm{O}_{2}$ and TEMPO radical shut down the reaction.

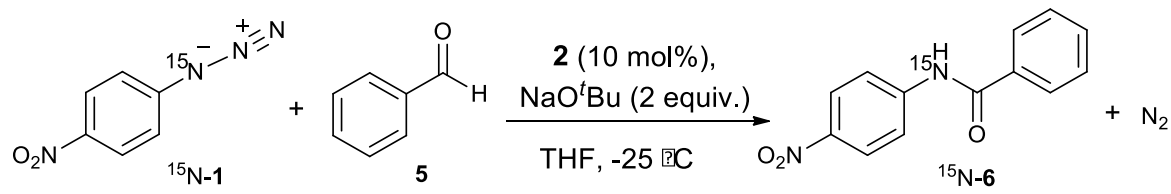

Scheme S2 Experiment carried out using ${ }^{15} \mathrm{~N}$-labelled $p$-nitroaniline ${ }^{15} \mathrm{~N}-1$ showed retention of the ${ }_{10}$ labeled ${ }^{15} \mathrm{~N}$ atom in the corresponding amide ${ }^{15} \mathrm{~N}-6$. 


\section{Materials and Methods}

${ }^{1} \mathrm{H}$ and ${ }^{13} \mathrm{C}-\mathrm{NMR}$ spectra were recorded on a Bruker AV (III) 400, Bruker AV 400, Bruker DPX 400, AV $3500\left(400 \mathrm{MHz}\right.$ or $500 \mathrm{MHz}\left({ }^{1} \mathrm{H}\right)$, and $100 \mathrm{MHz}$ or $\left.125 \mathrm{MHz}\left({ }^{13} \mathrm{C}\right)\right)$ spectrometers. Chemical shifts are expressed in parts per million ( $\mathrm{ppm})$ and the spectra calibrated to residual solvent ${ }_{5}$ signals of DMSO (2.54 ppm $\left({ }^{1} \mathrm{H}\right)$ and $\left.40.5 \mathrm{ppm}\left({ }^{13} \mathrm{C}\right)\right)$. Coupling constants are given in hertz $(\mathrm{Hz})$ and the following notations indicate the multiplicity of the signals: $s$ (singlet), $d$ (doublet), brd (broad doublet), dd (double doublet), t (triplet), tt (triple triplet), q (quartet), m (multiplet). High Resolution Mass Spectra were recorded on a VG micron Autospec or Bruker microTOF. Fourier Transform Infrared Spectroscopy (FT-IR) spectra were obtained using a Perkin Elmer 1600 series or Bruker ${ }_{10}$ Tensor 27 spectrometer. Melting points were recorded using a STUART SMP3 apparatus and are uncorrected. Thin layer chromatography was carried out on Merck pre-coated silica gel plates (60F254) and visualised using ultra violet light or $\mathrm{KMnO}_{4}$ solution. THF was freshly distilled from sodiumbenzophenone. Where necessary, reactions requiring anhydrous conditions were performed in dry solvents in flame dried or oven-dried apparatus under argon atmosphere. 


\section{General procedure:}

${ }^{t}$ BuONO (0.53 mmol, 1.05 eq.) was added to a mixture of the aniline ( $0.50 \mathrm{mmol}, 1.00$ eq.) and $\mathrm{TMSN}_{3}$ (0.53 mmol, 1.05 eq.) in THF $(2 \mathrm{~mL})$ at $0{ }^{\circ} \mathrm{C}$. The mixture was stirred until deemed complete (TLC). Then, the solution was cooled to $-25^{\circ} \mathrm{C}$ at which temperature the aldehyde $(0.75 \mathrm{mmol}, 1.50$ eq. $)$, ${ }_{5}$ thiazolium salt 2 (0.05 mmol, 0.10 eq.) and $\mathrm{NaO}^{t} \mathrm{Bu}(1.00 \mathrm{mmol}, 2$ eq.) were added sequentially. The resulting mixture was stirred until complete consumption of the azide (TLC). EtOAc (5 mL) and sat. $\mathrm{NaHSO}_{3(\mathrm{aq})}(5 \mathrm{~mL})$ were added and the layers separated. The organic layer was washed with $\mathrm{NaHSO}_{3(\mathrm{aq})}(5 \mathrm{~mL}), 1 \mathrm{M} \mathrm{HCl}_{(\mathrm{aq})}(5 \mathrm{~mL})$, dried $\left(\mathrm{MgSO}_{4}\right)$, filtered and evaporated under reduced pressure to give the crude product. Purification of the crude residue by flash column chromatography on silica ${ }_{10}$ with [95:5:1-80:20:1 petrol:EtOAc: $\left.\mathrm{Et}_{3} \mathrm{~N}\right]$ as eluent gave the corresponding amide.

\section{N-(4-nitrophenyl)benzamide (Table 2, Entry 1)}<smiles>O=C(Nc1ccc([N+](=O)[O-])cc1)c1ccccc1</smiles>

Yellow solid (0.139 g, 94 \%); $R_{\mathrm{f}}\left(70: 30\right.$ petrol-EtOAC) 0.3 ; mp $198-199{ }^{\circ} \mathrm{C}$ (lit., $\left.{ }^{1} 199-201{ }^{\circ} \mathrm{C}\right) ; \mathrm{IR}$ (FTIR, $\left.{ }_{15} \mathrm{CHCl}_{3}\right) v_{\max } \mathrm{cm}^{-1}: 3431(\mathrm{NH}), 1692(\mathrm{C}=\mathrm{O}), 1507\left(\mathrm{NO}_{2}\right), 1405,1345,1243,1113 ;{ }^{1} \mathrm{H}$ NMR (400 MHz, DMSO- $\left.d_{6}\right) \delta 10.8(\mathrm{~s}, 1 \mathrm{H}), 8.29-8.26(\mathrm{~m}, 2 \mathrm{H})$ 8.08-8.06 (m, 2H), 7.99-7.97 (m, 2H), 7.66-7.64 (m, $\left.1 \mathrm{H}\right)$, 7.59-7.55 (m, 2H); ${ }^{13} \mathrm{C}$ NMR (100 MHz, DMSO-d $\left.d_{6}\right) \delta 166.5$ (C), 145.7 (C), 142.7 (C), 134.2 (C), 132.4 (CH), $128.7(\mathrm{CH}), 128.1(\mathrm{CH}), 130.0(\mathrm{CH}), 120.9(\mathrm{CH}) ;{ }^{15} \mathrm{~N}$ NMR (100 MHz, DMSO-d $\left.d_{6}\right)-248.5$; HRMS ESI: calcd for $\mathrm{C}_{13} \mathrm{H}_{11} \mathrm{~N}_{2} \mathrm{O}_{3}[\mathrm{M}+\mathrm{H}]^{+}, 243.0764$; found, 243.0775; calcd for $\mathrm{C}_{13} \mathrm{H}_{10} \mathrm{NaN}_{2} \mathrm{O}_{3}[\mathrm{M}+\mathrm{Na}]^{+}, 265.0584$; ${ }_{20}$ found 265.0588 .

$\boldsymbol{N}$-(3-chloro-4-nitrophenyl)benzamide (Table 2, Entry 2) 
<smiles>O=C(Nc1ccc([N+](=O)[O-])cc1Cl)c1ccccc1</smiles>

Yellow solid $(0.117 \mathrm{~g}, 85 \%) ; R_{\mathrm{f}}\left(70: 30\right.$ petrol-EtOAC) $0.3 ; \mathrm{mp} 160{ }^{\circ} \mathrm{C}$ (lit., $\left.{ }^{2} 162-164{ }^{\circ} \mathrm{C}\right) ; \mathrm{IR}\left(\mathrm{FTIR}, \mathrm{CHCl}_{3}\right.$ ) $v_{\max } \mathrm{Cm}^{-1}: 3417(\mathrm{NH}), 1697$ (C=O), $1511\left(\mathrm{NO}_{2}\right), 1398,1345,1253,1121 ;{ }^{1} \mathrm{H}$ NMR (400 MHz, DMSO-d $\left.d_{6}\right)$ $\delta 10.3(\mathrm{~s}, 1 \mathrm{H}), 8.42(\mathrm{~d}, 1 \mathrm{H}, J=2.6 \mathrm{~Hz}), 8.29(\mathrm{dd}, 1 \mathrm{H}, J=8.9,2.6 \mathrm{~Hz}), 8.26(\mathrm{~d}, 1 \mathrm{H}, J=8.9 \mathrm{~Hz}), 8.03-7.99$ ${ }_{5}(\mathrm{~m}, 2 \mathrm{H}), 7.68-7.64(\mathrm{~m}, 1 \mathrm{H}), 7.60-7.55(\mathrm{~m}, 2 \mathrm{H}) ;{ }^{13} \mathrm{C}$ NMR (100 MHz, DMSO-d $\left.d_{6}\right) \delta 166.5(\mathrm{C}), 145.0$ (C), $141.3(\mathrm{C}), 133.4(\mathrm{C}), 132.4(\mathrm{CH}), 128.6(\mathrm{CH}), 128.1(\mathrm{C}), 127.9(\mathrm{CH}), 166.6(\mathrm{CH}), 124.9(\mathrm{CH}), 122.9(\mathrm{CH})$; HRMS ESI: calcd for $\mathrm{C}_{13} \mathrm{H}_{10} \mathrm{ClN}_{2} \mathrm{O}_{3}[\mathrm{M}+\mathrm{H}]^{+}, 277.0374$; found, 277.0373; calcd for $\mathrm{C}_{13} \mathrm{H}_{9} \mathrm{ClN}_{2} \mathrm{NaO}_{3}$ $[\mathrm{M}+\mathrm{Na}]^{+}, 299.0194 ;$ found 299.0189 .

${ }_{10} \boldsymbol{N}$-(4-nitro-2-(trifluoromethyl)phenyl)benzamide (Table 2, Entry 3)<smiles>O=C(Nc1ccc([N+](=O)[O-])cc1C(F)(F)F)c1ccccc1</smiles>

Yellow solid $(0.119 \mathrm{~g}, 77 \%), R_{\mathrm{f}}\left(70: 30\right.$ petrol-EtOAC) $0.3 ; \mathrm{mp} 158-161{ }^{\circ} \mathrm{C}$; IR $\left(\mathrm{FTIR}, \mathrm{CHCl}_{3}\right) v_{\max } \mathrm{cm}^{-1}$ : 3414 (NH), 1707 (C=O), $1534\left(\mathrm{NO}_{2}\right), 1508\left(\mathrm{NO}_{2}\right), 1398,1345,1161,1050 ;{ }^{1} \mathrm{H}$ NMR (500 MHz, DMSO$\left.d_{6}\right) \delta 10.42(\mathrm{~s}, 1 \mathrm{H}), 8.57(\mathrm{dd}, J=8.8,2.5 \mathrm{~Hz}, 1 \mathrm{H}), 8.52(\mathrm{~d}, J=2.5 \mathrm{~Hz}, 1 \mathrm{H}), 7.98-7.95(\mathrm{~m}, 3 \mathrm{H}), 7.67-7.63$ ${ }_{15}(\mathrm{~m}, 1 \mathrm{H}), 7.51-7.55(\mathrm{~m}, 2 \mathrm{H}) ;{ }^{13} \mathrm{C}$ NMR (125 MHz, DMSO-d $) \delta 166.4$ (C), 145.4 (C), 141.8 (C), 133.3 (C), $132.4(\mathrm{CH}), 131.8(\mathrm{CH}), 128.7(\mathrm{CH}), 128.0(\mathrm{CH}), 127.8(\mathrm{CH}), 126.3(\mathrm{C}, \mathrm{q}, J=33.1 \mathrm{~Hz}), 122.4(\mathrm{CH})$; HRMS ESI: calcd for $\mathrm{C}_{14} \mathrm{H}_{10} \mathrm{~F}_{3} \mathrm{~N}_{2} \mathrm{O}_{3}[\mathrm{M}+\mathrm{H}]^{+}, 311.0638$; found, 311.0633; calcd for $\mathrm{C}_{14} \mathrm{H}_{9} \mathrm{~F}_{3} \mathrm{~N}_{2} \mathrm{NaO}_{3}[\mathrm{M}+\mathrm{Na}]^{+}$, 333.0457; found 333.0453 .

N-(4-nitro-3-(trifluoromethyl)phenyl)benzamide (Table 2, Entry 4) 
<smiles>O=C(Nc1ccc([N+](=O)[O-])c(C(F)(F)F)c1)c1ccccc1</smiles>

Yellow solid (0.130 g, 84\%); $R_{\mathrm{f}}\left(70: 30\right.$ petrol-EtOAc) 0.3; mp $129-131{ }^{\circ} \mathrm{C}$ (lit., $\left.{ }^{3} 129{ }^{\circ} \mathrm{C}\right)$; IR (FTIR, $\mathrm{CHCl}_{3}$ ) $v_{\max } \mathrm{cm}^{-1}: 3431(\mathrm{NH}), 1694$ (C=O), $1520\left(\mathrm{NO}_{2}\right), 1415,1322,1250$ (CF), 1163 (CF); ${ }^{1} \mathrm{H}$ NMR (500 MHz, DMSO-d $d_{6} \delta 11.0(\mathrm{~s}, 1 \mathrm{H}), 8.49(\mathrm{~d}, J=2.2 \mathrm{~Hz}, 1 \mathrm{H}), 8.35$ (dd, $\left.J=9.0,2.2 \mathrm{~Hz}, 1 \mathrm{H}\right), 8.26(\mathrm{~d}, J=9.0 \mathrm{~Hz}, 1 \mathrm{H})$, s 8.02-7.99 (m, 2H), 7.66-7.64 (m, 1H), 7.60-7.57 (m, 2H); ${ }^{13} \mathrm{C}$ NMR (125 MHz, DMSO-d 6 ) $170.0(\mathrm{C})$, 144.4 (C), 142.1 (C), 134.2 (C), 133.0 (C), 129.7 (CH), 129.1 (CH), $128.4(\mathrm{CH}), 128.1(\mathrm{CH}), 123.7(\mathrm{CH})$, $123.2(\mathrm{C}, \mathrm{q}, J=33.2 \mathrm{~Hz}), 118.8(\mathrm{CH})$; HRMS ESI: calcd for $\mathrm{C}_{14} \mathrm{H}_{9} \mathrm{~F}_{3} \mathrm{~N}_{2} \mathrm{NaO}_{3}[\mathrm{M}+\mathrm{Na}]^{+}, 333.0457$; found 333.0450 .

Methoxy-N-(4-nitrophenyl)benzamide (Table 2, Entry 5)<smiles>COc1ccc(C(=O)Nc2ccc([N+](=O)[O-])cc2)cc1</smiles>

Yellow solid (0.124 g, $91 \%) ; R_{\mathrm{f}}\left(70: 30\right.$ petrol-EtOAC) $0.3 ; \mathrm{mp} 183-185{ }^{\circ} \mathrm{C}$ (lit., $\left.{ }^{4} 184-185{ }^{\circ} \mathrm{C}\right) ; \mathrm{IR}(\mathrm{FTIR}$, $\left.\mathrm{CHCl}_{3}\right) v_{\max } \mathrm{cm}^{-1}: 3435(\mathrm{NH}), 1685$ (C=O), $1504\left(\mathrm{NO}_{2}\right), 1345,1240,1113,1030 ;{ }^{1} \mathrm{H} \mathrm{NMR}(400 \mathrm{MHz}$, DMSO- $\left.d_{6}\right) \delta 10.6(\mathrm{~s}, 1 \mathrm{H}), 8.26-8.24(\mathrm{~m}, 2 \mathrm{H}) 8.07-8.04(\mathrm{~m}, 2 \mathrm{H}), 8.01-7.98(\mathrm{~m}, 2 \mathrm{H}), 7.11-7.08(\mathrm{~m}, 2 \mathrm{H})$, $3.31(\mathrm{~s}, 3 \mathrm{H}) ;{ }^{13} \mathrm{C}$ NMR (100 MHz, DMSO-d $\left.d_{6}\right) \delta 165.9$ (C), 162.8 (C), 146.2 (C), 142.7 (C), 130.4 (CH), $126.5(\mathrm{C}), 125.2(\mathrm{CH}), 120.1(\mathrm{CH}), 114.2(\mathrm{CH}), 55.9\left(\mathrm{CH}_{3}\right)$; HRMS ESI: calcd for $\mathrm{C}_{14} \mathrm{H}_{13} \mathrm{~N}_{2} \mathrm{O}_{4}[\mathrm{M}+\mathrm{H}]^{+}$, 273.0870; found, 273.0869; calcd for $\mathrm{C}_{14} \mathrm{H}_{12} \mathrm{NaN}_{2} \mathrm{O}_{4}[\mathrm{M}+\mathrm{Na}]^{+}$, 295.0689; found 295.0689.

3,5-Dimethoxy- $\mathrm{N}$-(4-nitrophenyl)benzamide (Table 2, Entry 6) 


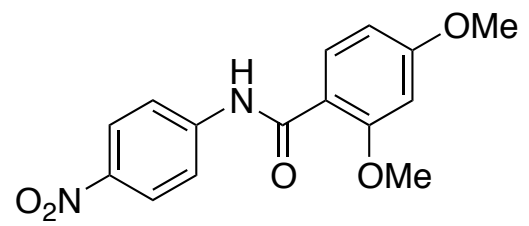

Yellow solid (0.119 g, 79\%), $R_{\mathrm{f}}\left(70: 30\right.$ petrol-EtOAC) 0.2; $\mathrm{mp} 198-199^{\circ} \mathrm{C} ; \mathrm{IR}\left(\mathrm{FTIR}, \mathrm{CHCl}_{3}\right) v_{\max } \mathrm{cm}^{-1}: 3356$ (NH), 1674 (C=O), 1604, $1548\left(\mathrm{NO}_{2}\right), 1342,1249,1027 ;{ }^{1} \mathrm{H}$ NMR (400 MHz, DMSO-d 6 ) $\delta 10.45$ (s, 1H), 8.26-8.22 (m, 2H), 8.01-7.98 (m, 2H), $7.72(\mathrm{~d}, J=8.6 \mathrm{~Hz}, 1 \mathrm{H}), 6.72(\mathrm{~d}, J=2.3 \mathrm{~Hz}, 1 \mathrm{H}), 6.68(\mathrm{dd}, J=8.6$, s $2.31 \mathrm{H}), 3.94(\mathrm{~s}, 3 \mathrm{H}), 3.85(\mathrm{~s}, 3 \mathrm{H}) ;{ }^{13} \mathrm{C}$ NMR (100 MHz, DMSO-d $)_{6} \delta 164.5$ (C), 163.4 (C), 158.5 (C), $145.2(\mathrm{C}), 142.2(\mathrm{C}), 132.0(\mathrm{CH}), 124.9(\mathrm{CH}), 119.4(\mathrm{CH}), 115.8(\mathrm{C}), 105.9(\mathrm{CH}), 95.6(\mathrm{CH}), 56.7\left(\mathrm{CH}_{3}\right)$, $56.1\left(\mathrm{CH}_{3}\right)$; HRMS ESI: calcd for $\mathrm{C}_{15} \mathrm{H}_{15} \mathrm{~N}_{2} \mathrm{O}_{5}[\mathrm{M}+\mathrm{H}]^{+}$, 303.0975; found, 303.0973; calcd for $\mathrm{C}_{15} \mathrm{H}_{14} \mathrm{~N}_{2} \mathrm{NaO}_{5}[\mathrm{M}+\mathrm{Na}]^{+}, 325.0795$; found 325.0792 .

\section{${ }_{10}$ 4-Methoxy-N-(4-nitro-3-(trifluoromethyl)phenyl)benzamide (Table 2, Entry 7)}<smiles>COc1ccc(C(=O)Nc2ccc([N+](=O)[O-])c(C(F)(F)F)c2)cc1</smiles>

Yellow solid $(0.138 \mathrm{~g}, 89 \%) ; R_{\mathrm{f}}\left(70: 30\right.$ petrol-EtOAC) $0.3 ; \mathrm{mp} 110-113{ }^{\circ} \mathrm{C}$ (lit., $\left.{ }^{3} 112-114{ }^{\circ} \mathrm{C}\right) ; \mathrm{IR}(\mathrm{FTIR}$, $\left.\mathrm{CHCl}_{3}\right) v_{\max } \mathrm{cm}^{-1}: 3436(\mathrm{NH}), 1686$ (C=O), $1508\left(\mathrm{NO}_{2}\right), 1250$ (CF), 1175 (CF), 1097, 1030; ${ }^{1} \mathrm{H}$ NMR (500 $\left.{ }_{5} \mathrm{MHz}, \mathrm{DMSO}-d_{6}\right) \delta 10.80(\mathrm{~s}, 1 \mathrm{H}), 8.48(\mathrm{~d}, J=2.3 \mathrm{~Hz}, 1 \mathrm{H}), 8.35(\mathrm{dd}, J=9.0,2.3 \mathrm{~Hz}, 1 \mathrm{H}), 8.23(\mathrm{~d}, J=9.0$ $\mathrm{Hz}, 1 \mathrm{H}), 8.02-7.99(\mathrm{~m}, 2 \mathrm{H}), 7.12-7.10(\mathrm{~m}, 2 \mathrm{H}), 3.32(\mathrm{~s}, 3 \mathrm{H}) ;{ }^{13} \mathrm{C}$ NMR $\left(125 \mathrm{MHz}, \mathrm{DMSO}-d_{6}\right) \delta 165.7(\mathrm{C})$, 162.7 (C), 144.3 (C), 141.4 (C), 130.1 (CH), 127.7 (CH), 125.6 (C), 123.0 (CH), 122.5 (C, q, J = 33.2 Hz), $121.1(\mathrm{C}), 118.2(\mathrm{CH}), 133.9(\mathrm{CH}), 55.6\left(\mathrm{CH}_{3}\right)$; HRMS ESI: calcd for $\mathrm{C}_{15} \mathrm{H}_{12} \mathrm{~F}_{3} \mathrm{~N}_{2} \mathrm{O}_{4}[\mathrm{M}+\mathrm{H}]^{+}, 341.0744$; found, 341.0743; calcd for $\mathrm{C}_{15} \mathrm{H}_{11} \mathrm{~F}_{3} \mathrm{~N}_{2} \mathrm{NaO}_{4}[\mathrm{M}+\mathrm{Na}]^{+}$, 363.0563; found 363.0568 .

4-Nitro-N-(4-nitrophenyl) benzamide (Table 2, Entry 8) 


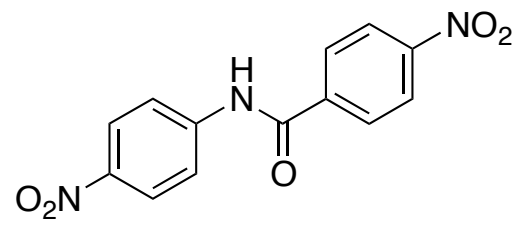

Yellow solid (0.052 g, 38\%), $R_{\mathrm{f}}\left(70: 30\right.$ petrol-EtOAC) $0.2 ; \mathrm{mp} 266-270{ }^{\circ} \mathrm{C}$ (lit., $\left.{ }^{5} 267-269{ }^{\circ} \mathrm{C}\right)$; IR (FTIR, $\left.\mathrm{CHCl}_{3}\right) v_{\max } \mathrm{cm}^{-1}: 3693(\mathrm{NH}), 1600(\mathrm{C}=\mathrm{O}), 1530\left(\mathrm{NO}_{2}\right), 1511\left(\mathrm{NO}_{2}\right), 1346,1241,1113 ;{ }^{1} \mathrm{H} \mathrm{NMR}(500$ $\left.\mathrm{MHz}, \mathrm{DMSO}-d_{6}\right) \delta 11.11(\mathrm{~s}, 1 \mathrm{H}), 8.42-8.41(\mathrm{~m}, 2 \mathrm{H}), 8.39-8.38(\mathrm{~m}, 2 \mathrm{H}), 8.23-8.22(\mathrm{~m}, 2 \mathrm{H}), 8.09-8.05$ ${ }_{5}(\mathrm{~m}, 2 \mathrm{H}) ;{ }^{13} \mathrm{C}$ NMR (125 MHz, DMSO-d $\left.{ }_{6}\right) 165.2$ (C), 149.0 (C), 145.5 (C), 143.3 (C), 140,4 (C), 130.0 (CH), $125.4(\mathrm{CH}), 124.1(\mathrm{CH}), 120.6(\mathrm{CH})$; HRMS ESI: calcd for $\mathrm{C}_{13} \mathrm{H}_{10} \mathrm{~N}_{3} \mathrm{O}_{5}[\mathrm{M}+\mathrm{H}]^{+}, 288.2351$; found, 288.9213; calcd for $\mathrm{C}_{13} \mathrm{H}_{9} \mathrm{~N}_{3} \mathrm{NaO}_{5}[\mathrm{M}+\mathrm{Na}]^{+}, 310.2169$; found 310.0425.

\section{$\mathbf{N}$-(4-nitrophenyl)thiophene-2-carboxamide (Table 2, Entry 9)}

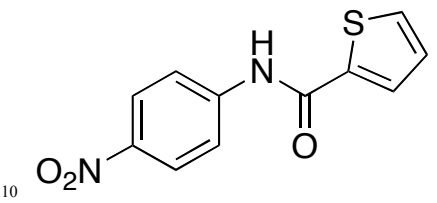

Yellow solid (0.102 g, 82\%), $R_{\mathrm{f}}$ (70:30 petrol:EtOAC) 0.3; mp 221-223 ${ }^{\circ} \mathrm{C}$ (lit., ${ }^{6} 222-224{ }^{\circ} \mathrm{C}$ ); IR (FTIR, $\left.\mathrm{CHCl}_{3}\right) \mathrm{v}_{\max } \mathrm{cm}^{-1}: 3430(\mathrm{NH}), 1673$ (amide), 1600, $1506\left(\mathrm{NO}_{2}\right), 1344,1245,1113 ;{ }^{1} \mathrm{H} \mathrm{NMR}(500 \mathrm{MHz}$, DMSO- $\left.d_{6}\right) \delta 10.75(\mathrm{~s}, 1 \mathrm{H}), 8.29-8.25(\mathrm{~m}, 2 \mathrm{H}), 8.10(\mathrm{dd}, J=3.8,1.1 \mathrm{~Hz}, 1 \mathrm{H}), 8.04-8.00(\mathrm{~m}, 2 \mathrm{H}), 7.95$ $(\mathrm{dd}, J=5.0,1.1 \mathrm{~Hz}, 1 \mathrm{H}), 7.27(\mathrm{dd}, J=5.0,3.8 \mathrm{~Hz}, 1 \mathrm{H}) ;{ }^{13} \mathrm{C}$ NMR $\left(125 \mathrm{MHz}, \mathrm{DMSO}-d_{6}\right) \delta 160.5(\mathrm{C}), 145.2$ ${ }_{15}(\mathrm{C}), 142.5(\mathrm{C}), 139.1(\mathrm{C}), 133.1(\mathrm{CH}), 130.3(\mathrm{CH}), 128.3(\mathrm{CH}), 124.9(\mathrm{CH}), 119.8(\mathrm{CH})$; HRMS ESI: calcd for $\mathrm{C}_{11} \mathrm{H}_{9} \mathrm{~N}_{2} \mathrm{O}_{3} \mathrm{~S}[\mathrm{M}+\mathrm{H}]^{+}$, 249.0328; found, 249.0315; calcd for $\mathrm{C}_{11} \mathrm{H}_{8} \mathrm{~N}_{2} \mathrm{NaO}_{3} \mathrm{~S}[\mathrm{M}+\mathrm{Na}]^{+}, 271.0148$; found 271.0140.

$\boldsymbol{N}$-(4-nitrophenyl)-2-phenylpropanamide (Table 2, Entry 10)

${ }_{25}$<smiles>CC(C(=O)Nc1ccc([N+](=O)[O-])cc1)c1ccccc1</smiles> 
Yellow solid (0.127 g, 94\%), $R_{\mathrm{f}}\left(70: 30\right.$ petrol:EtOAC) 0.3; mp 168-169 ${ }^{\circ} \mathrm{C} ; \mathrm{IR}\left(\mathrm{FTIR}, \mathrm{CHCl}_{3}\right) \mathrm{v}_{\max } \mathrm{cm}^{-1}$ : $3401(\mathrm{NH}), 1702$ (C=O), 1599, $1533\left(\mathrm{NO}_{2}\right), 1506\left(\mathrm{NO}_{2}\right), 1344,1249,1177,1114 ;{ }^{1} \mathrm{H} \mathrm{NMR}(400 \mathrm{MHz}$, DMSO- $\left.d_{6}\right) \delta 10.65(\mathrm{~s}, 1 \mathrm{H}), 8.22-8.18(\mathrm{~m}, 2 \mathrm{H}), 7.86-7.82(\mathrm{~m}, 2 \mathrm{H}), 7.40-7.32(\mathrm{~m}, 4 \mathrm{H}), 7.26-7.22(\mathrm{~m}$, $\left.{ }_{5} 1 \mathrm{H}\right), 3.89$ (q, $\left.J=7.0 \mathrm{~Hz}, 1 \mathrm{H}\right), 1.43(\mathrm{~d}, J=7.0 \mathrm{~Hz}, 3 \mathrm{H}) ;{ }^{13} \mathrm{C}$ NMR (100 MHz, DMSO-d $\left.d_{6}\right) \delta 173.2$ (C), 145.3 (C), 142.1 (C), 141.2 (C), $128.5(\mathrm{CH}), 127.3(\mathrm{CH}), 126.9(\mathrm{CH}), 124.9(\mathrm{CH}), 118.9(\mathrm{CH}), 46.2(\mathrm{CH}), 18.6$ $\left(\mathrm{CH}_{3}\right)$; HRMS ESI: calcd for $\mathrm{C}_{15} \mathrm{H}_{15} \mathrm{~N}_{2} \mathrm{O}_{3}[\mathrm{M}+\mathrm{H}]^{+}$, 271.1077; found, 271.1070; calcd for $\mathrm{C}_{15} \mathrm{H}_{14} \mathrm{~N}_{2} \mathrm{NaO}_{3}$ $[\mathrm{M}+\mathrm{Na}]^{+}, 293.0897$; found 293.0901.

${ }_{10} \boldsymbol{N}$-(4-nitrophenyl)cyclohexanecarboxamide (Table 2, Entry 11)<smiles>O=C(Nc1ccc([N+](=O)[O-])cc1)C1CCCCC1</smiles>

Colourless solid (0.109 g, 88\%); $R_{\mathrm{f}}$ (70:30 petrol-EtOAC) 0.4 ; mp $160-163^{\circ} \mathrm{C}$ (lit.. $\left.{ }^{7} 162-163{ }^{\circ} \mathrm{C}\right)$; IR (FTIR, $\left.\left.\mathrm{CHCl}_{3}\right) v_{\max } \mathrm{cm}^{-1}: 3429(\mathrm{NH}), 1702(\mathrm{C}=\mathrm{O})\right), 1505\left(\mathrm{NO}_{2}\right), 1345,1246,1163,1114 ;{ }^{1} \mathrm{H}$ NMR $(400 \mathrm{MHz}$, DMSO- $\left.d_{6}\right) \delta 10.4(\mathrm{~s}, 1 \mathrm{H}), 8.21-8.17(\mathrm{~m}, 2 \mathrm{H}), 7.86-7.83(\mathrm{~m}, 2 \mathrm{H}), 2.83(\mathrm{tt}, J=11.5,3.5 \mathrm{~Hz}, 1 \mathrm{H}), 1.84-$ ${ }_{15} 1.81(\mathrm{~m}, 2 \mathrm{H}), 1.78-1.74(\mathrm{~m}, 2 \mathrm{H}), 1.66-1.64(\mathrm{~m}, 1 \mathrm{H}), 1.45-1.16(\mathrm{~m}, 5 \mathrm{H}) ;{ }^{13} \mathrm{C}$ NMR $\left(100 \mathrm{MHz}, \mathrm{DMSO}-d_{6}\right)$ $\delta 175.2(\mathrm{C}), 145.7$ (C), $141.9(\mathrm{C}), 124.9(\mathrm{CH}), 118.6(\mathrm{CH}), 44.9(\mathrm{CH}), 28.9\left(\mathrm{CH}_{2}\right), 25.3\left(\mathrm{CH}_{2}\right), 25.1\left(\mathrm{CH}_{2}\right)$; HRMS ESI: calcd for $\mathrm{C}_{13} \mathrm{H}_{16} \mathrm{NaN}_{2} \mathrm{O}_{3}[\mathrm{M}+\mathrm{Na}]^{+}, 271.1053$; found 271.1055.

$\boldsymbol{N}$-(4-nitrophenyl)cyclopropanecarboxamide (Table 2, Entry 12) ${ }^{9}$<smiles>O=C(Nc1ccc([N+](=O)[O-])cc1)C1CC1</smiles> 
Yellow solid (0.102 g, 78\%), $R_{\mathrm{f}}$ (70:30 petrol:EtOAC) $0.4 ; \mathrm{mp} 145-147{ }^{\circ} \mathrm{C}$ (lit., ${ }^{8} 183-185{ }^{\circ} \mathrm{C}$ ); IR (FTIR, $\left.\mathrm{CHCl}_{3}\right) \mathrm{V}_{\max } \mathrm{cm}^{-1}: 3430(\mathrm{NH}), 1701$ (C=O), $1507\left(\mathrm{NO}_{2}\right), 1343\left(\mathrm{NO}_{2}\right), 1159,1035,953,853 ;{ }^{1} \mathrm{H} \mathrm{NMR}(400$ MHz, DMSO- $\left.d_{6}\right) \delta 10.81(\mathrm{~s}, 1 \mathrm{H}), 8.22-8.18(\mathrm{~m}, 2 \mathrm{H}), 7.85-7.81(\mathrm{~m}, 2 \mathrm{H}), 2.50$ (app quintet, $J=1.8 \mathrm{~Hz}$, $1 \mathrm{H}), 0.88-0.86(\mathrm{~m}, 4 \mathrm{H}) ;{ }^{13} \mathrm{C}$ NMR (100 MHz, DMSO-d $\left.d_{6}\right) 172.7$ (C), 144.4 (C), 141.9 (C), 125.0 (CH), s $118.6(\mathrm{CH}), 14.8(\mathrm{CH}), 7.9\left(\mathrm{CH}_{2}\right)$; HRMS ESI: calcd for $\mathrm{C}_{10} \mathrm{H}_{10} \mathrm{~N}_{2} \mathrm{NaO}_{3}[\mathrm{M}+\mathrm{Na}]^{+}, 229.0584$; found, 229.0581.

N-(2-chloro-4-nitrophenyl)cyclohexanecarboxamide (Table 2, Entry 13)<smiles>O=C(Nc1ccc([N+](=O)[O-])cc1Cl)C1CCCCC1</smiles>

${ }_{10}$ Colourless solid $(0.130 \mathrm{~g}, 92 \%) ; R_{\mathrm{f}}\left(70: 30\right.$ petrol-EtOAc) $0.4 ; \mathrm{mp} 156-158{ }^{\circ} \mathrm{C} ; \mathrm{IR}\left(\mathrm{FTIR}, \mathrm{CHCl}_{3}\right) v_{\max } \mathrm{cm}^{-1}$ : $3692(\mathrm{NH}), 1619$ (C=O), $1524\left(\mathrm{NO}_{2}\right), 1443,1241,1025,930 ;{ }^{1} \mathrm{H}$ NMR (400 MHz, DMSO-d 6 ) $\delta 9.69$ (s, $1 \mathrm{H}), 8.34-8.34(\mathrm{~m}, 1 \mathrm{H}), 8.19-8.19(\mathrm{~m}, 2 \mathrm{H}), 2.64(\mathrm{tt}, J=11.4,3.4 \mathrm{~Hz}, 1 \mathrm{H}), 1.86-1.83(\mathrm{~m}, 2 \mathrm{H}), 1.76-1.74$ $(\mathrm{m}, 2 \mathrm{H}), 1.67-1.64(\mathrm{~m}, 1 \mathrm{H}), 1.45-1.16(\mathrm{~m}, 5 \mathrm{H}) ;{ }^{13} \mathrm{C}$ NMR $\left(100 \mathrm{MHz}, \mathrm{DMSO}-d_{6}\right) \delta 175.2(\mathrm{C}), 143.1(\mathrm{C})$, $141.3(\mathrm{C}), 124.9(\mathrm{CH}), 124.8(\mathrm{C}), 124.1(\mathrm{CH}), 123.0(\mathrm{CH}), 44.1(\mathrm{CH}), 29.1\left(\mathrm{CH}_{2}\right), 25.3\left(\mathrm{CH}_{2}\right), 25.1\left(\mathrm{CH}_{2}\right)$; ${ }_{15} \mathrm{HRMS}$ ESI: calcd for $\mathrm{C}_{13} \mathrm{H}_{16} \mathrm{ClN}_{2} \mathrm{O}_{3}[\mathrm{M}+\mathrm{H}]^{+}, 283.0844$; found, 283.0834; calcd for $\mathrm{C}_{13} \mathrm{H}_{15} \mathrm{ClN}_{2} \mathrm{NaO}_{3}$ $[\mathrm{M}+\mathrm{Na}]^{+}, 305.0663$; found 305.0653 .

\section{$\mathbf{N}$-(4-nitro-2-(trifluoromethyl)phenyl)cyclohexanecarboxamide (Table 2, Entry 14)}<smiles>O=C(Nc1ccc([N+](=O)[O-])cc1C(F)(F)F)C1CCCCC1</smiles>

${ }_{20}$ Colourless solid $(0.131 \mathrm{~g}, 83 \%): R_{\mathrm{f}}\left(70: 30\right.$ petrol-EtOAC) $0.4 ; \mathrm{mp} 142-145^{\circ} \mathrm{C}$; IR (FTIR, $\left.\mathrm{CHCl}_{3}\right) v_{\max } \mathrm{cm}^{-1}$ : $3428(\mathrm{NH}), 1706$ (C=O), $1517\left(\mathrm{NO}_{2}\right), 1320,1275$ (CF), 1163 (CF), 1077; ${ }^{1} \mathrm{H}$ NMR (500 MHz, DMSO-d 6 ) $\delta$ $9.72(\mathrm{~s}, 1 \mathrm{H}), 8.49(\mathrm{dd}, J=8.9,2.6 \mathrm{~Hz}, 1 \mathrm{H}), 8.44(\mathrm{~d}, J=2.6 \mathrm{~Hz}, 1 \mathrm{H}), 7.89(\mathrm{~d}, J=8.9 \mathrm{~Hz}, 1 \mathrm{H}), 2.55(\mathrm{tt}, J=$ 11.5, $3.4 \mathrm{~Hz}, 1 \mathrm{H}), 1.85-1.82(\mathrm{~m}, 2 \mathrm{H}), 1.78-1.74(\mathrm{~m}, 2 \mathrm{H}), 1.66-1.62(\mathrm{~m}, 1 \mathrm{H}), 1.44-1.16(\mathrm{~m}, 5 \mathrm{H}) ;{ }^{13} \mathrm{C}$ NMR (125 MHz, DMSO-d $\left.d_{6}\right) \delta 175.2$ (C), 144.2 (C), 141.6 (C), $130.1(\mathrm{CH}), 127.8(\mathrm{CH}), 123.5(\mathrm{C}, \mathrm{q}, J=$ $\left.{ }_{25} 31.3 \mathrm{~Hz}\right), 122.2(\mathrm{CH}), 121.4(\mathrm{C}), 43.8(\mathrm{CH}), 28.9\left(\mathrm{CH}_{2}\right), 25.4\left(\mathrm{CH}_{2}\right), 25.1\left(\mathrm{CH}_{2}\right)$; HRMS ESI: calcd for 
$\mathrm{C}_{14} \mathrm{H}_{16} \mathrm{~F}_{3} \mathrm{~N}_{2} \mathrm{O}_{3}[\mathrm{M}+\mathrm{H}]^{+}$, 317.1108; found, 317.1094; calcd for $\mathrm{C}_{14} \mathrm{H}_{15} \mathrm{~F}_{3} \mathrm{~N}_{2} \mathrm{NaO}_{3}[\mathrm{M}+\mathrm{Na}]^{+}$, 339.0927; found 339.0914 .

$\mathbf{N - ( 4 - n i t r o - 3 - ( t r i f l u o r o m e t h y l ) p h e n y l ) c y c l o h e x a n e c a r b o x a m i d e ~ ( T a b l e ~ 2 , ~ E n t r y ~ 1 5 ) ~}$<smiles>O=C(Nc1ccc([N+](=O)[O-])c(C(F)(F)F)c1)C1CCCCC1</smiles>

Colourless solid (0.138 g, 87\%); $R_{\mathrm{f}}\left(70: 30\right.$ petrol-EtOAC) $0.4 ; \mathrm{mp} 128-130{ }^{\circ} \mathrm{C}$ (lit., $\left.{ }^{3} 129-131{ }^{\circ} \mathrm{C}\right)$; IR (FTIR, $\mathrm{CHCl}_{3}$ ) $v_{\max } \mathrm{cm}^{-1}: 3415(\mathrm{NH}), 1706$ (C=O), $1517\left(\mathrm{NO}_{2}\right), 1380,1225$ (CF), 1142 (CF), 1068; ${ }^{1} \mathrm{H}$ NMR (500 MHz, DMSO-d 6 ) $\delta 10.62(\mathrm{~s}, 1 \mathrm{H}), 8.29(\mathrm{~d}, J=2.2 \mathrm{~Hz}, 1 \mathrm{H}), 8.17(\mathrm{~d}, J=8.9 \mathrm{~Hz}, 1 \mathrm{H}), 8.04(\mathrm{dd}, J=8.9,2.2$ $\mathrm{Hz}, 1 \mathrm{H}), 2.36(\mathrm{tt}, J=11.4,3.4 \mathrm{~Hz}, 2 \mathrm{H}), 1.85-1.82(\mathrm{~m}, 2 \mathrm{H}), 1.77-173(\mathrm{~m}, 2 \mathrm{H}), 1.66-1.63(\mathrm{~m}, 1 \mathrm{H}), 1.45-$ ${ }_{10} 1.15(\mathrm{~m}, 5 \mathrm{H}) ;{ }^{13} \mathrm{C}$ NMR (125 MHz, DMSO-d 6 ) $\delta 175.6$ (C), 144.2 (C), 141.1 (C), 127.8 (CH), 122.9 (C, q, J $=31.3 \mathrm{~Hz}), 122.0(\mathrm{CH}), 121.0(\mathrm{C}), 117.1(\mathrm{CH}), 45.1(\mathrm{CH}), 28.9\left(\mathrm{CH}_{2}\right), 25.3\left(\mathrm{CH}_{2}\right), 25.1\left(\mathrm{CH}_{2}\right)$; HRMS ESI: calcd for $\mathrm{C}_{14} \mathrm{H}_{16} \mathrm{~F}_{3} \mathrm{~N}_{2} \mathrm{O}_{3}[\mathrm{M}+\mathrm{H}]^{+}$, 317.1108; found, 317.1095; calcd for $\mathrm{C}_{14} \mathrm{H}_{15} \mathrm{~F}_{3} \mathrm{~N}_{2} \mathrm{NaO}_{3}[\mathrm{M}+\mathrm{Na}]^{+}$, 339.0927; found 339.0919.

is 2-((Z)-3-benzyl-4-methyl-2-((E)-(4-nitrophenyl)triaz-2-en-1-ylidene)-2,3-dihydrothiazol-5-yl) ethanol (7)

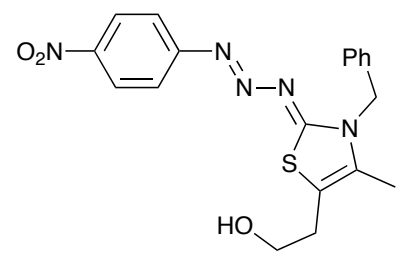

1-azido-4-nitrobenzene $1(50.0 \mathrm{mg}, 0.305 \mathrm{mmol})$ and the thiazolium salt $2(82.0 \mathrm{mg}, 0.305 \mathrm{mmol})$ were dissolved in THF $(1 \mathrm{~mL})$ and the corresponding mixture was cooled to $-78{ }^{\circ} \mathrm{C}$. NaH $(30.5 \mathrm{mg}, 60 \%$ ${ }_{20} \mathrm{~W} / \mathrm{w}$ mineral oil, $0.761 \mathrm{mmol}$ ) was added to the mixture in one portion. The reaction mixture was allowed to warm to room temperature, at which point a bright red colour appeared. The reaction was stirred at room temperature until complete (TLC, $5 \mathrm{~h}$ ). The reaction mixture was then poured onto saturated ammonium chloride solution $(5 \mathrm{~mL})$ and the products extracted with ethyl acetate $(3 \mathrm{x}$ $5 \mathrm{~mL}$ ). The combined organic extracts were dried over anhydrous magnesium sulfate, filtered and the 
solvents removed in vacuo. The resulting residue was finally subjected to flash column chromatography (eluting with ethyl acetate) to deliver the product as a bright red solid (98.0 mg, 81\%), $R_{\mathrm{f}}$ (EtOAc) 0.2; IR (FTIR, $\left.\mathrm{CHCl}_{3}\right) v_{\max } \mathrm{cm}^{-1}: 3108(\mathrm{OH}), 1520\left(\mathrm{NO}_{2}\right), 1428(\mathrm{~N}=\mathrm{N}), 1327,1134,1106$; ${ }^{1} \mathrm{H}$ NMR $\left(400 \mathrm{MHz}, \mathrm{DMSO}-d_{6}\right) \delta 8.28(\mathrm{~d}, J=8.9 \mathrm{~Hz}, 2 \mathrm{H}), 7.64(\mathrm{~d}, J=8.9 \mathrm{~Hz}, 2 \mathrm{H}), 7.41-7.37(\mathrm{~m}, 2 \mathrm{H})$, s 7.33-7.30 (m, 1H), 7.26-7.24 (m, 2H), $5.42(\mathrm{~s}, 2 \mathrm{H}), 4.93(\mathrm{t}, J=5.7 \mathrm{~Hz}, 1 \mathrm{H}), 3.59(\operatorname{app~q}, J=5.7 \mathrm{~Hz}, 2 \mathrm{H})$, $2.76(t, J=5.7 \mathrm{~Hz}, 2 \mathrm{H}), 2.16(\mathrm{~s}, 3 \mathrm{H}) ;{ }^{13} \mathrm{C}$ NMR (100 MHz, DMSO- $\left.d_{6}\right) \delta 176.8(\mathrm{C}), 155.6(\mathrm{C}), 145.4(\mathrm{C})$, $135.8(\mathrm{C}), 133.1(\mathrm{C}), 128.9(\mathrm{CH}), 127.7(\mathrm{CH}), 126.5(\mathrm{CH}), 125.0(\mathrm{CH}), 121.7(\mathrm{CH}), 116.5(\mathrm{C}), 60.5\left(\mathrm{CH}_{2}\right)$, $48.6\left(\mathrm{CH}_{2}\right), 29.7\left(\mathrm{CH}_{2}\right), 11.2\left(\mathrm{CH}_{3}\right)$; HRMS ESI: calcd for $\mathrm{C}_{19} \mathrm{H}_{20} \mathrm{~N}_{5} \mathrm{O}_{3} \mathrm{~S}[\mathrm{M}+\mathrm{H}]^{+}, 398.1281$; found, 398.1291; calcd for $\mathrm{C}_{19} \mathrm{H}_{19} \mathrm{~N}_{5} \mathrm{NaO}_{3} \mathrm{~S}[\mathrm{M}+\mathrm{Na}]^{+}$, 420.1101; found, 420.1122. 


\section{References}

[1] P. Sharma, A. D. Moorhouse and J. E. Moses, Synlett, 16, 2011, 2384-2386.

[2] G. Chattopadhyay, S. Chakraborty and C. Saha, Synth. Commum., 38, 2008, 4068-4075.

[3] A. W. Humm and M. R. Schneider, Archiv der Pharmazie, 321, 1988, 419-422.

[4] T. J. Broxton, C. J. Carolane and L. W. Deady, Aust. J. Chem., 28, 1975, 451-454.

[5] W. -P. Hu, H. -S. Yu, Y. -R. Chen, Y. -M. Tsai, Y. -K. Chen, C. -C. Liao, L. -S. Chang and J. -J. Wang, Bioorg. Medicinal Chem., 16, 2008, 5295-5302.

[6] H. Zimmer, R. E. Koenigkrammer, R. L. Cepulis and D. M. Nene., J. Org. Chem., 45, 2018-2020.

[7] R. M. Herbst, C. W. Roberts, H. T. F. Givens, and E. K. Harvill, J. Org. Chem., 17, 1952, 262-269.

${ }_{10}$ [8] Y. -H. Yang and M. Shi, J. Org. Chem., 70, 2005, 8645-8648. 
NMR spectra

2-((Z)-3-benzyl-4-methyl-2-((E)-(4-nitrophenyl)triaz-2-en-1-ylidene)-2,3-dihydrothiazol-5-yl) ethanol (SI1) 
2/10/2012 14:32:40

UserlD j_bur SamplelD jb1254 SupervisorlD moses Lab Phone No. 13540 Slot Number 35

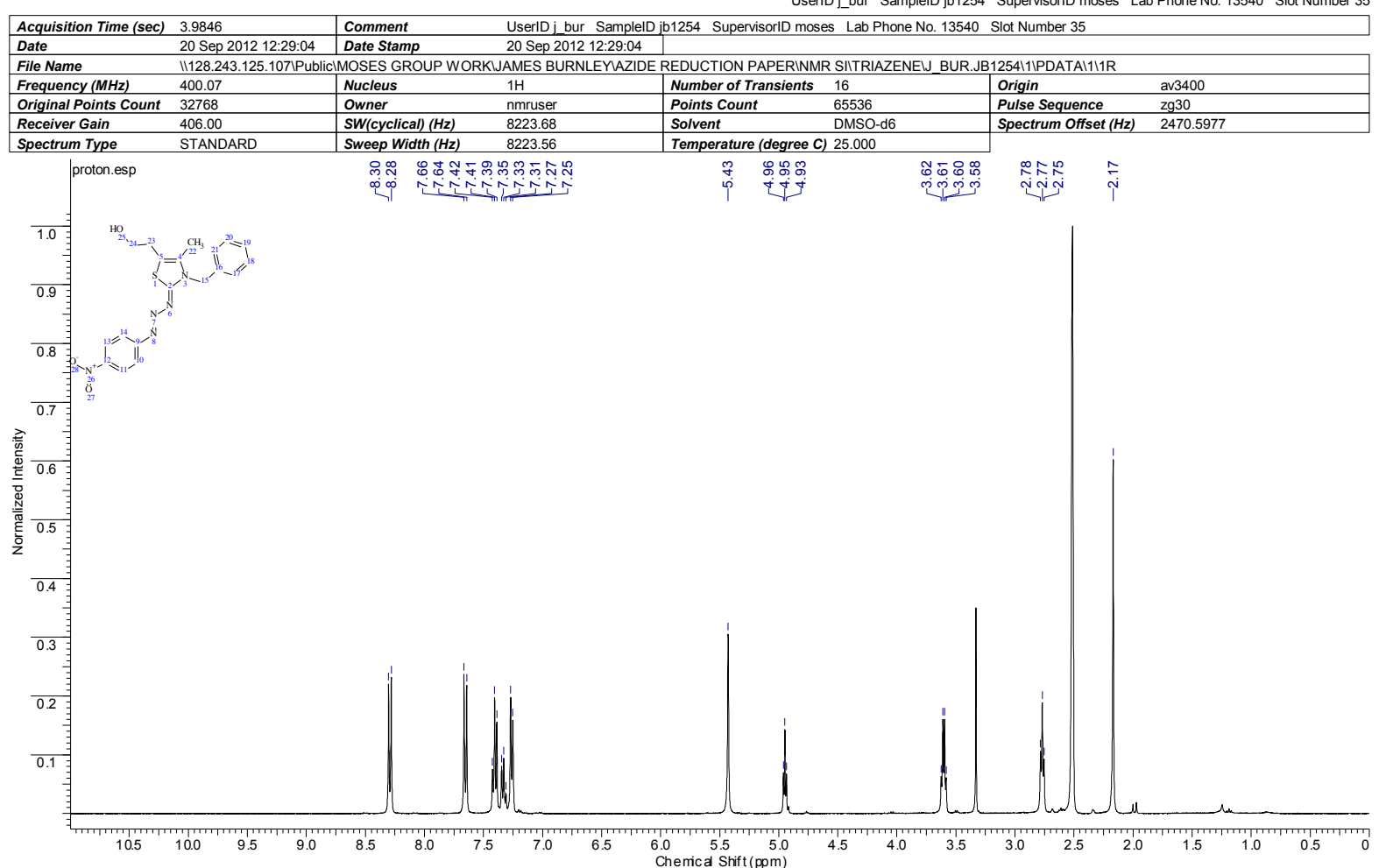

R:IMoses Group WorklJames Burnleylazide reduction paperINMR SIltriazenelproton.esp

UserlD j_bur SamplelD jb1254 SupervisorlD moses Lab Phone No. 13540 Slot Number 12

\section{\begin{tabular}{|l|lr|}
\hline Formula $\mathrm{C}_{19} \mathrm{H}_{19} \mathrm{~N}_{5} \mathrm{O}_{3} \mathrm{~S}$ & FW & 397.4509 \\
\hline
\end{tabular}}

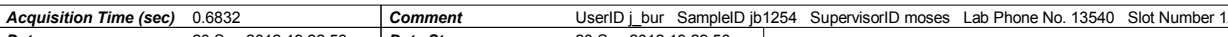

\begin{tabular}{|ll|ll|l}
\hline Date & 20 Sep 2012 19:22:56 & Date Stamp & 20 Sep 2012 19:22:56 & \\
\hline File Name & I1128.243.125.107|PUBLICIMOSES GROUP WORKIJAMES BURNLEYAZIDE REDUCTION PAPERINMR SIITRIAZENEIJ_BUR.JB1254 CARBONI3IPDATAI111R
\end{tabular}

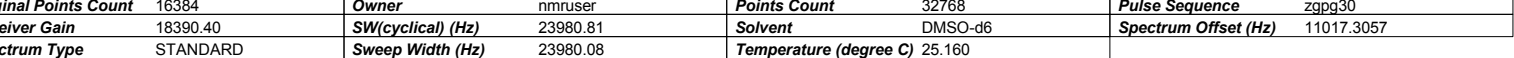

\begin{tabular}{|l} 
Spectrum Type STANDARD \\
\hline
\end{tabular}

Sweep Width $(\mathrm{Hz}) \quad 23980.08$

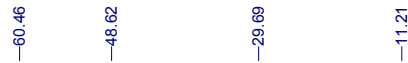

। । ।

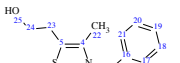

in
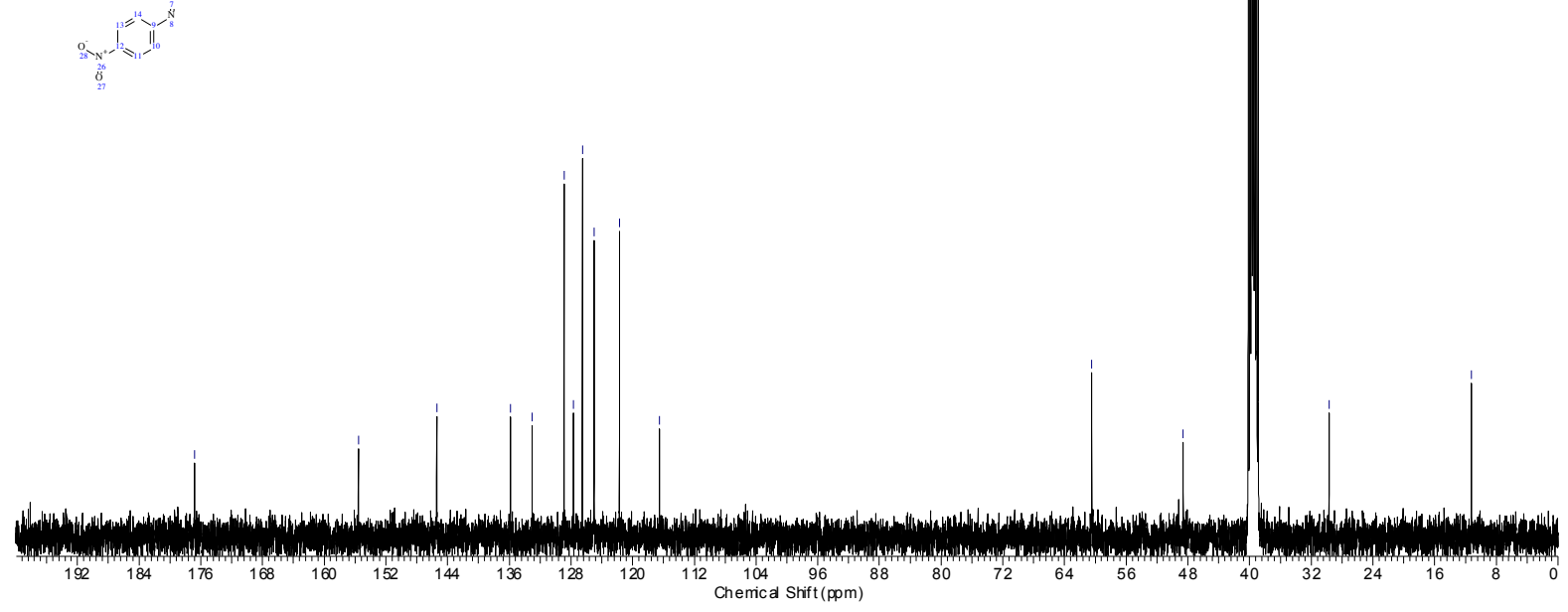

II128.243.125.107/PublicIMoses Group WorklJames Burnleylazide reduction paperINMR SIltriazeneltraizene.esp

$\mathbf{N}$-(4-nitrophenyl)benzamide (Table 2, Entry 1) 

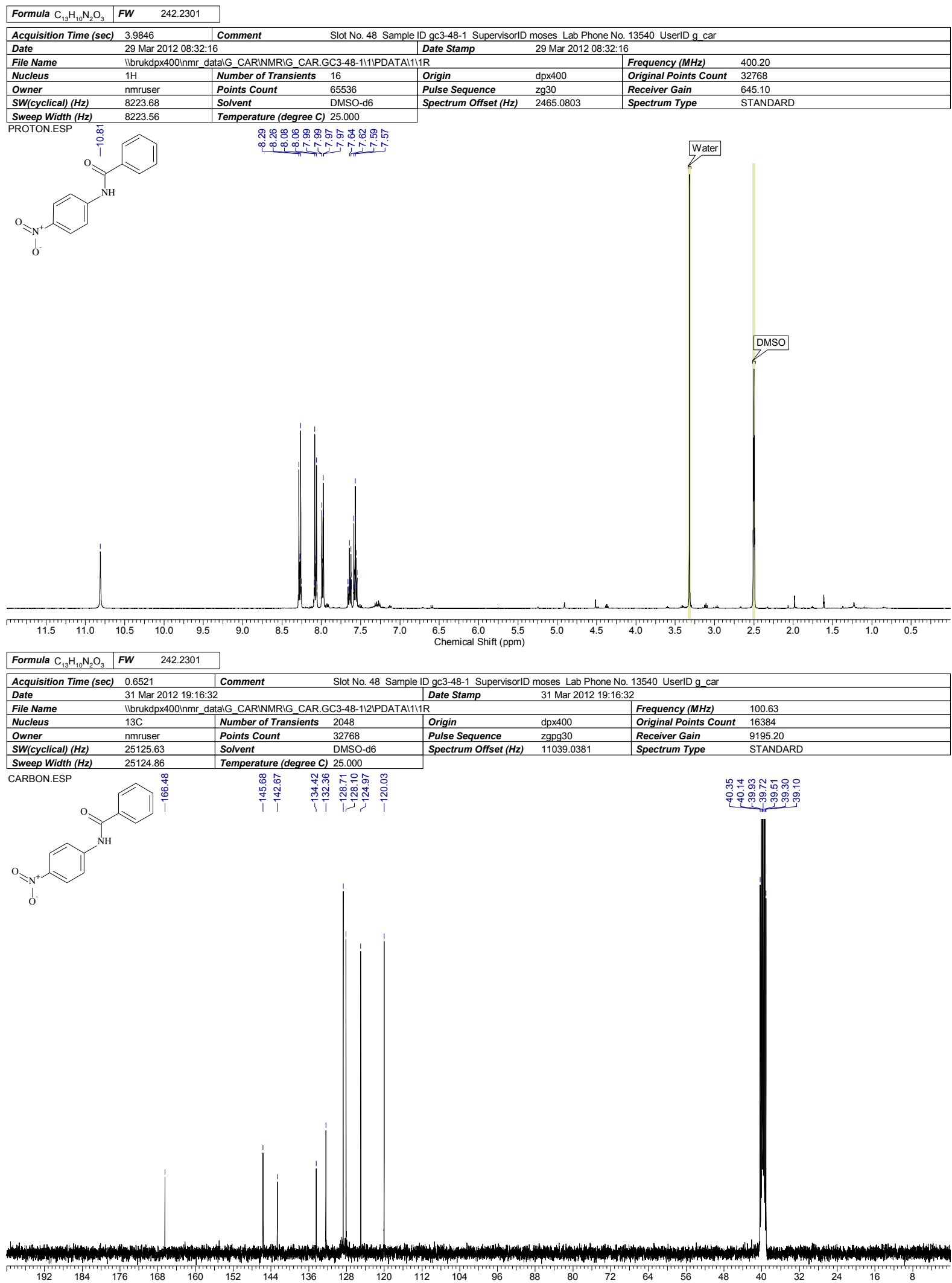

$\mathbf{N}$-(3-chloro-4-nitrophenyl)benzamide (Table 2, Entry 2) 


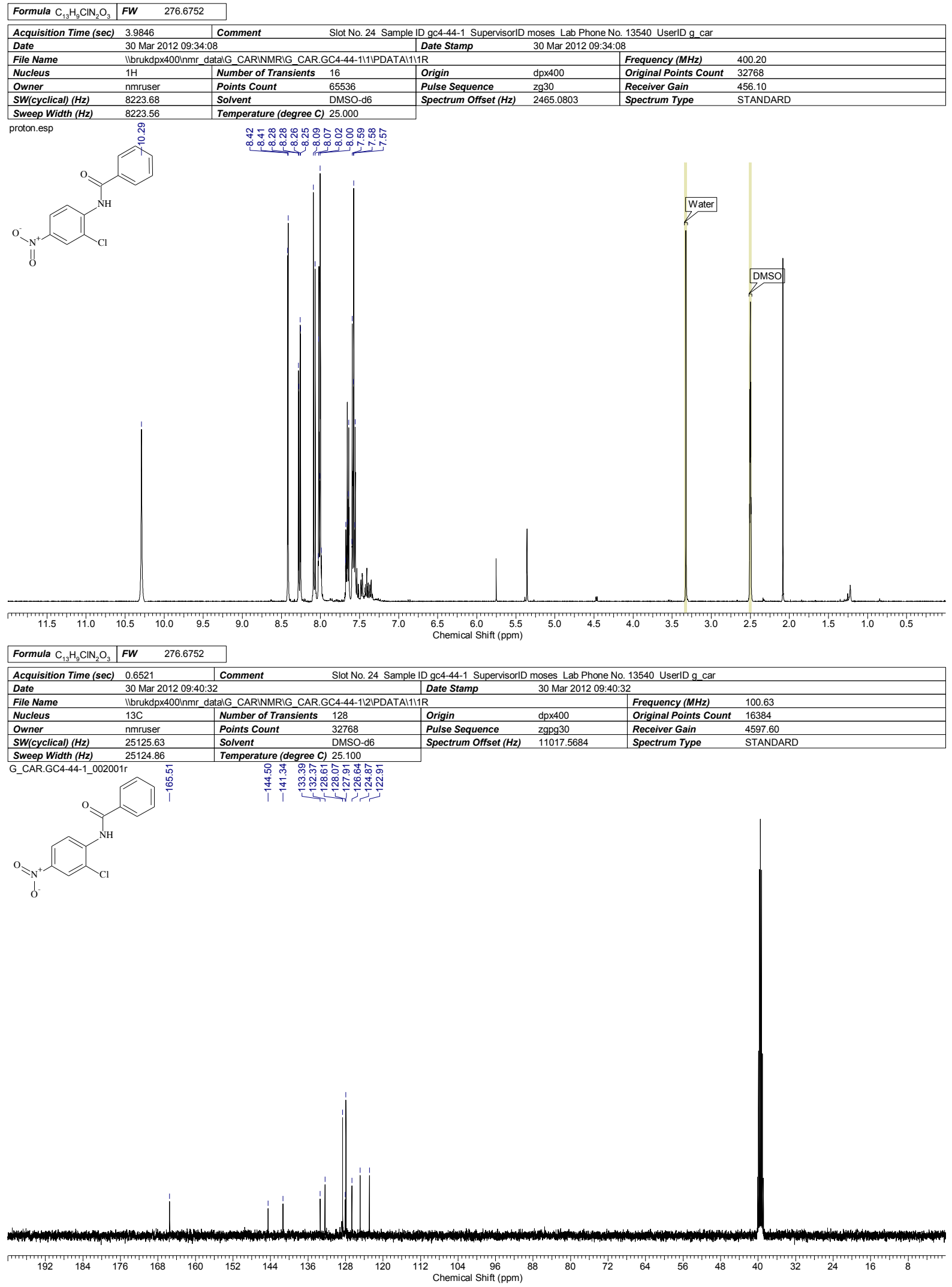

$\mathbf{N}$-(4-nitro-2-(trifluoromethyl)phenyl)benzamide (Table 2, Entry 3) 

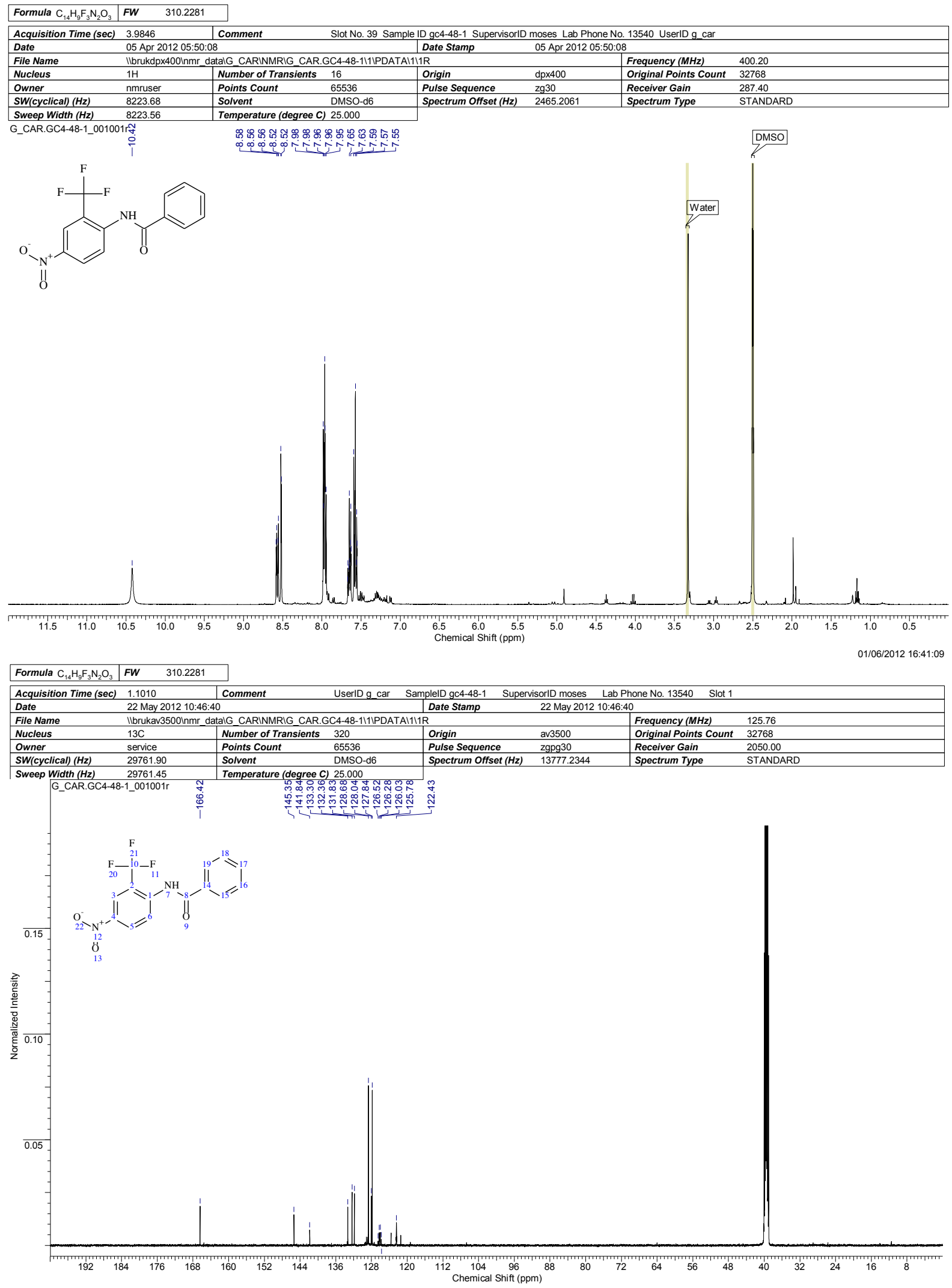

$\mathbf{N}$-(4-nitro-3-(trifluoromethyl)phenyl)benzamide (Table 2, Entry 4) 


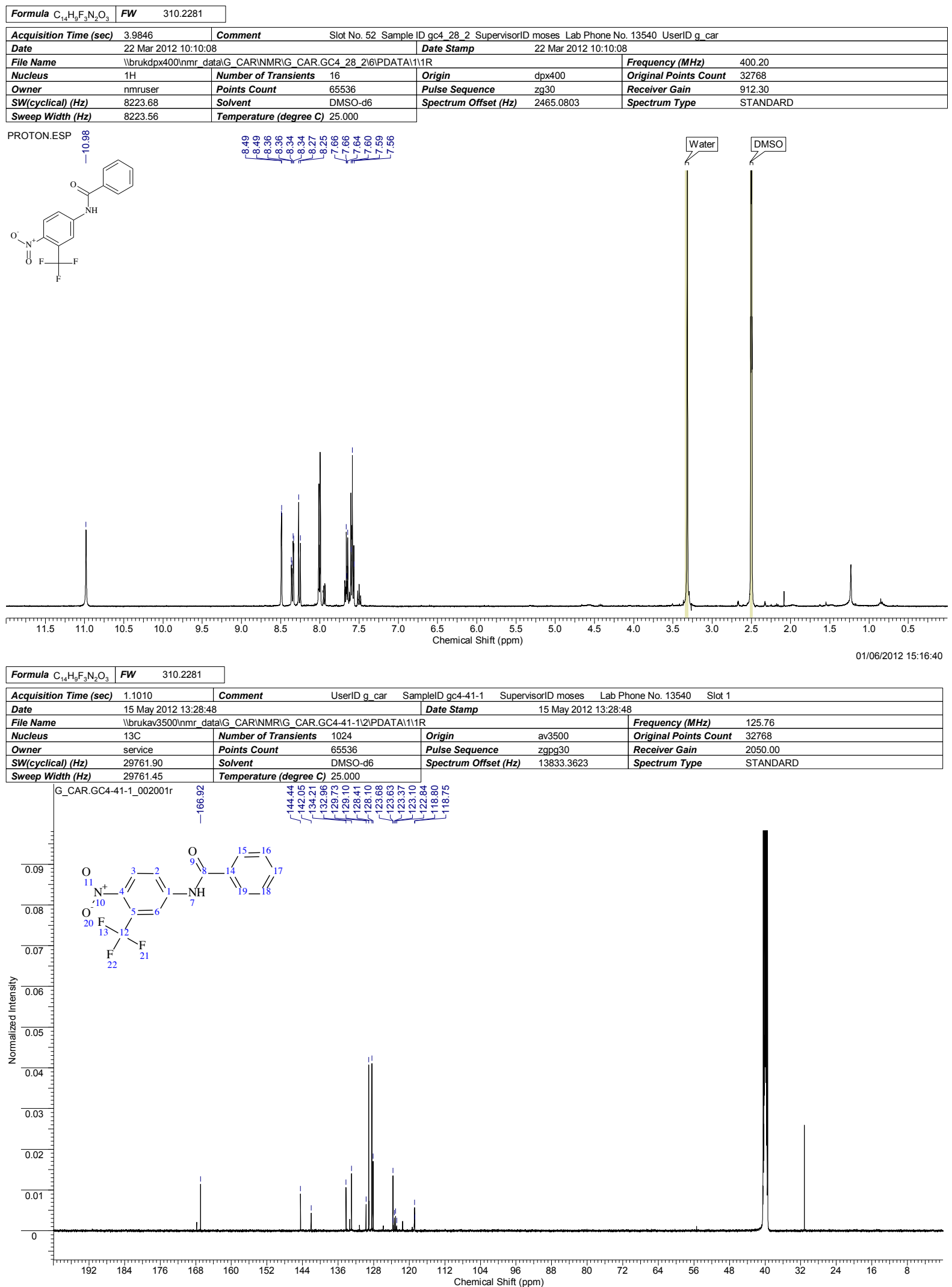

Methoxy-N-(4-nitrophenyl)benzamide (Table 2, Entry 5) 


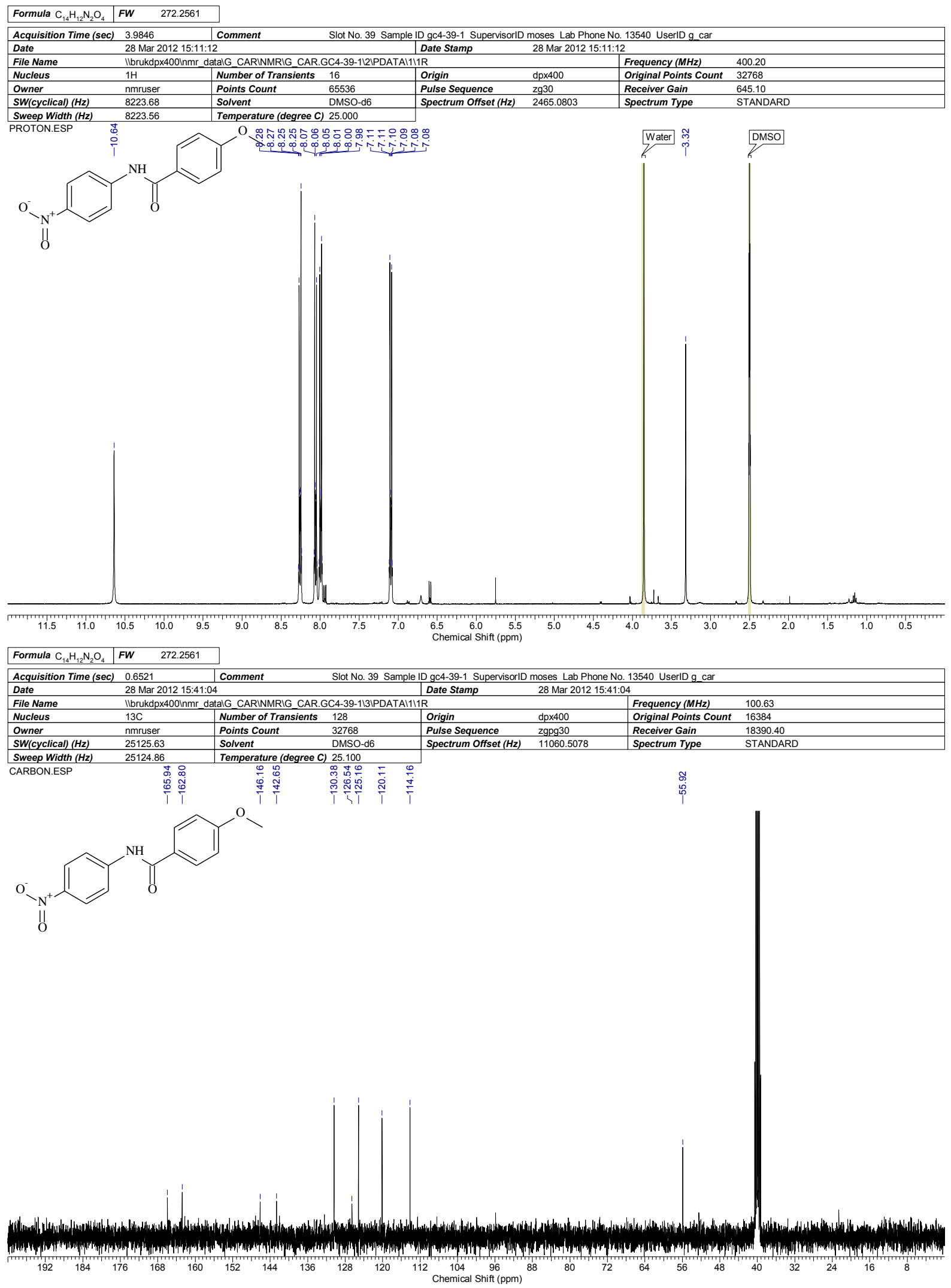

3,5-Dimethoxy- $\mathbf{N}$-(4-nitrophenyl)benzamide (Table 2, Entry 6) 


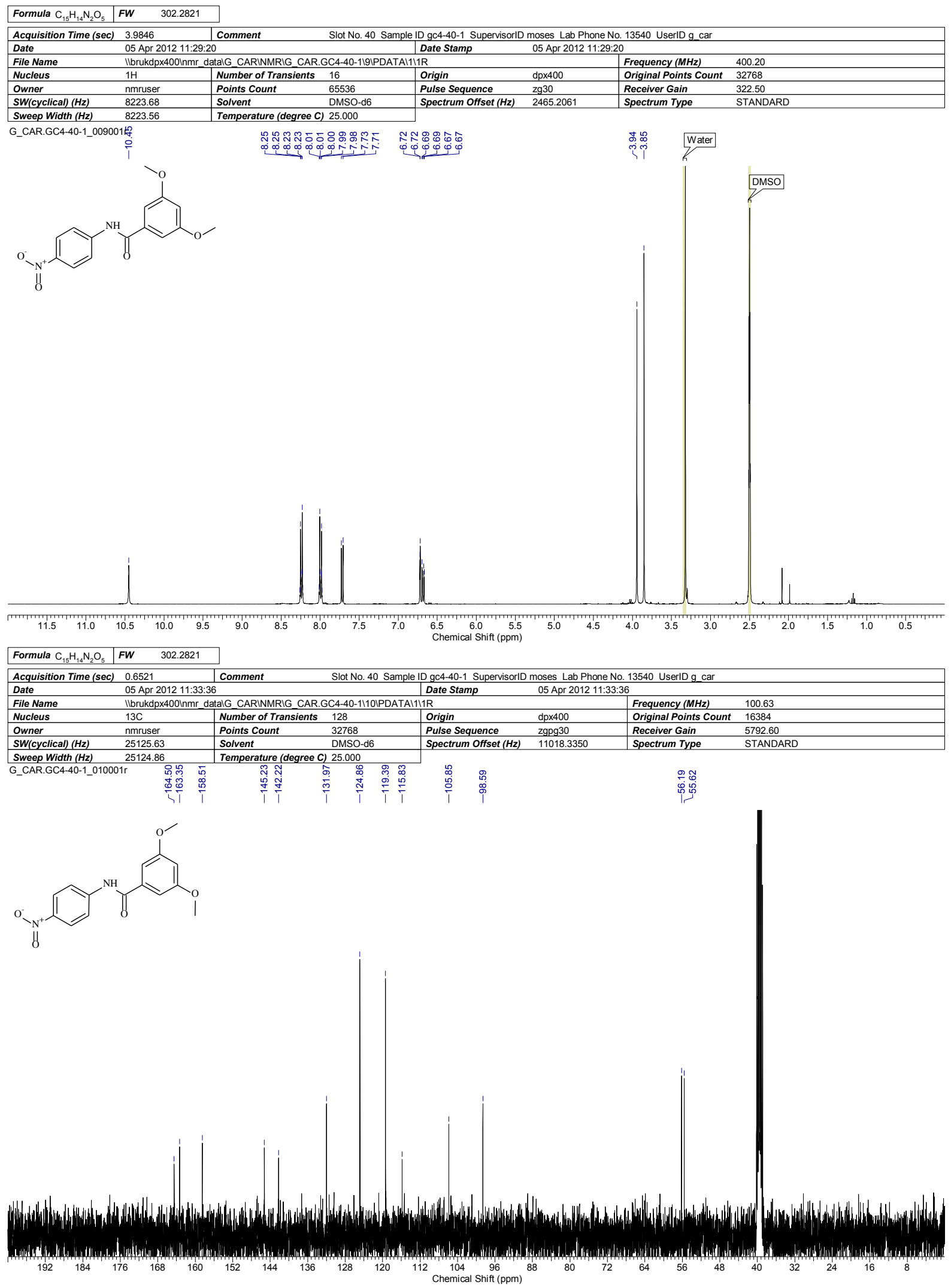

4-Methoxy-N-(4-nitro-3-(trifluoromethyl)phenyl)benzamide (Table 2, Entry 7) 


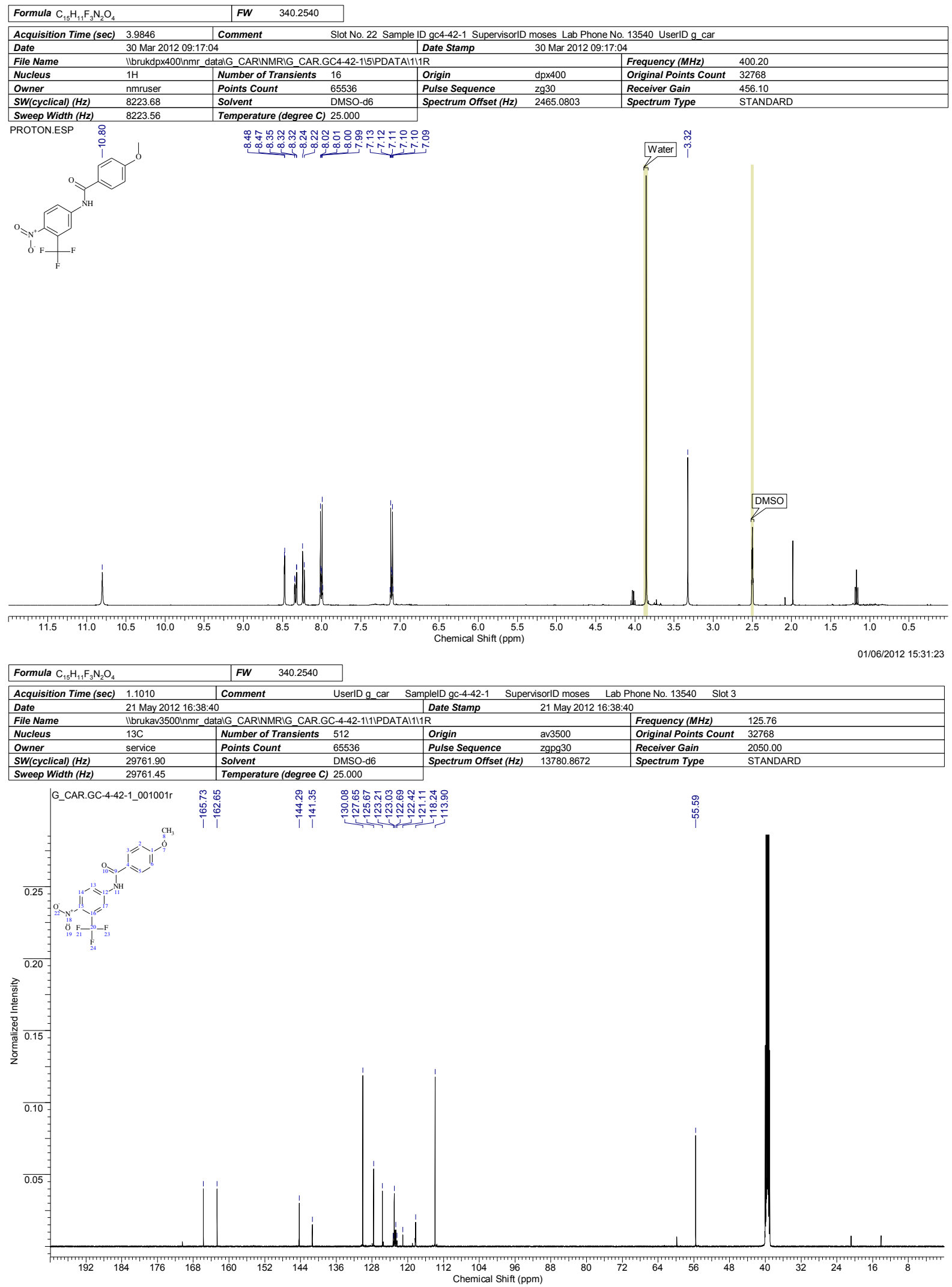

4-Nitro-N-(4-nitrophenyl) benzamide (Table 2, Entry 8) 


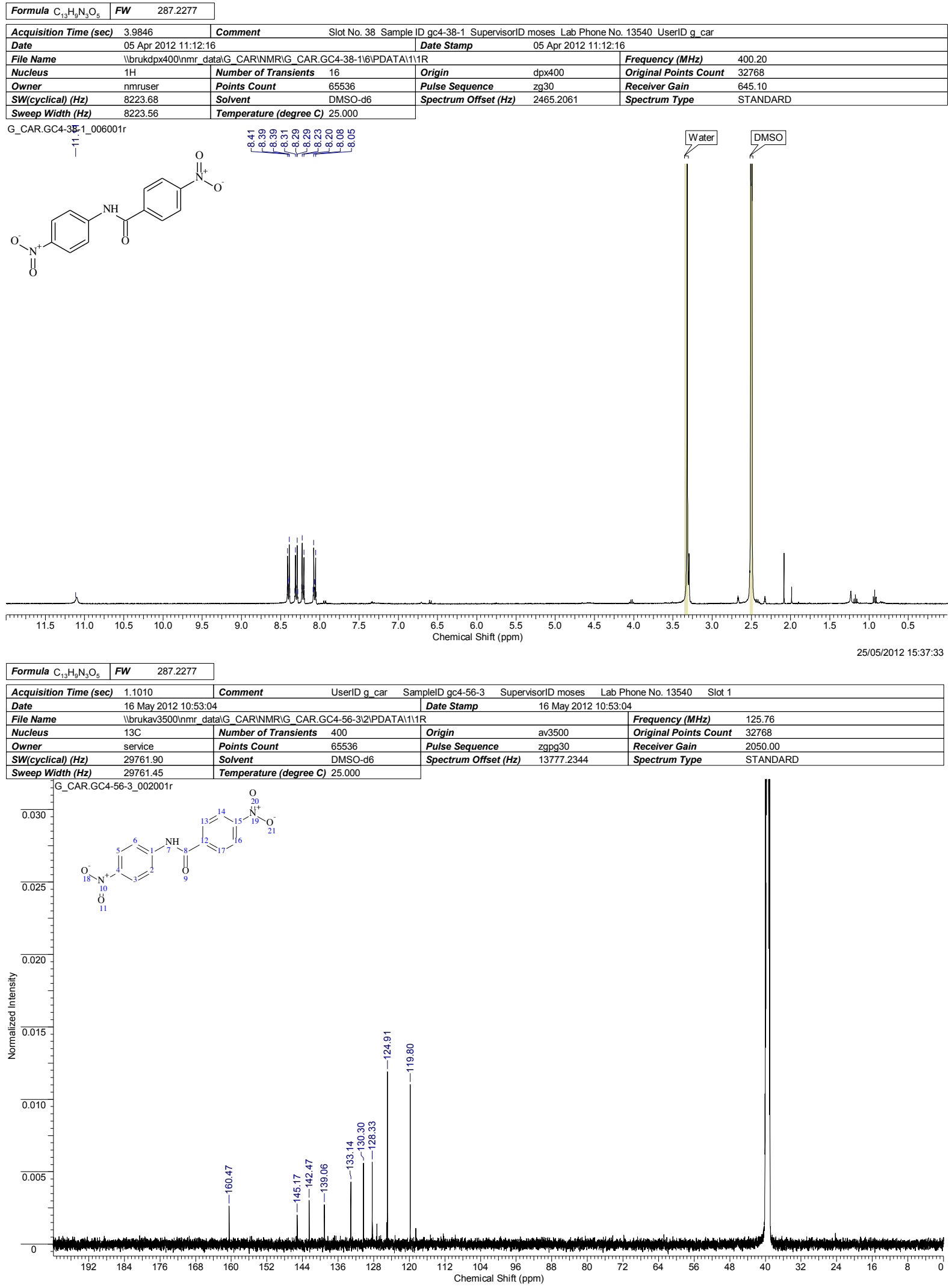

$\mathbf{N}$-(4-nitrophenyl)thiophene-2-carboxamide (Table 2, Entry 9) 

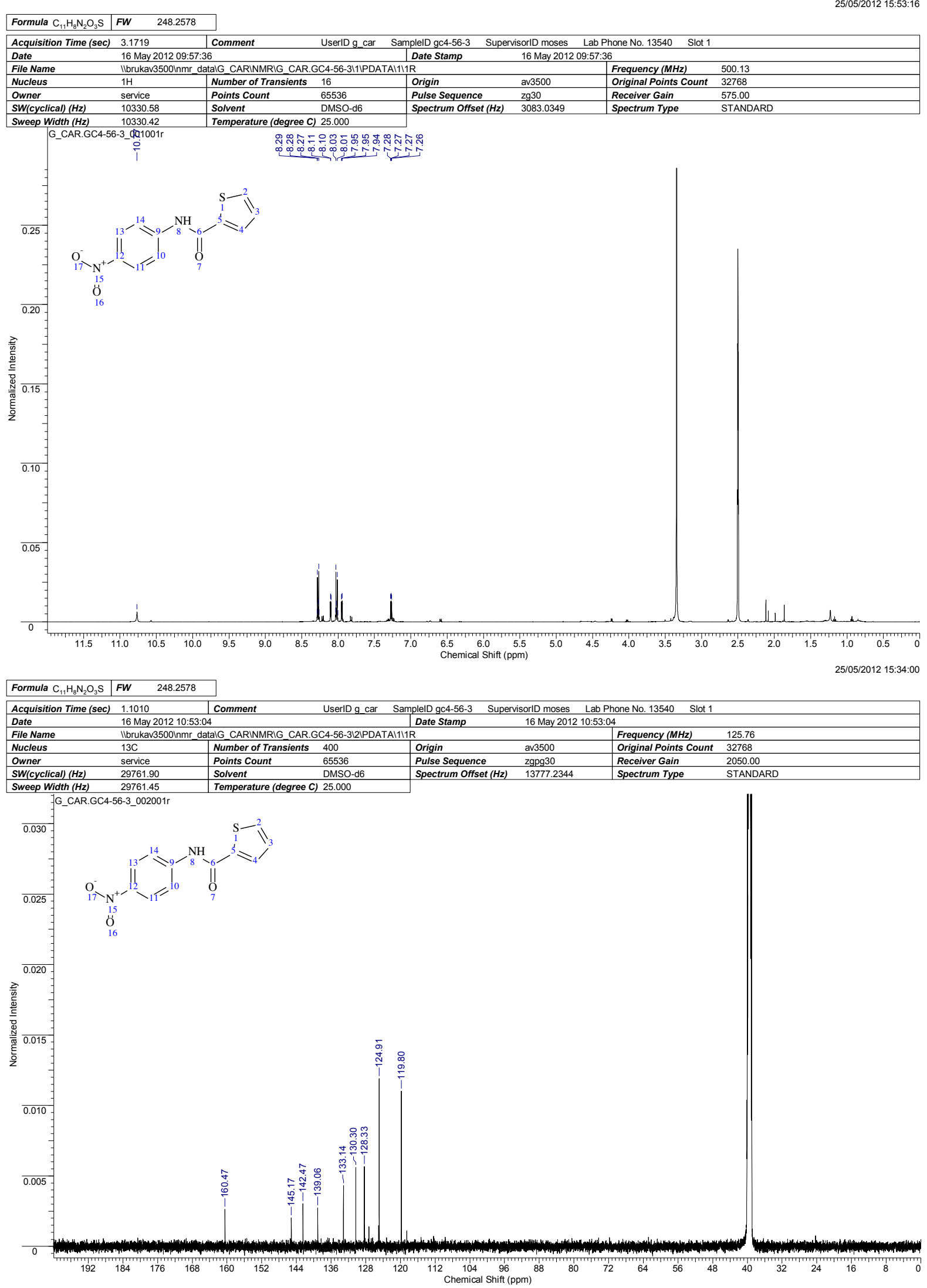

$\boldsymbol{N}$-(4-nitrophenyl)-2-phenylpropanamide (Table 2, Entry 10) 
\begin{tabular}{|l|lr|}
\hline Formula $\mathrm{C}_{15} \mathrm{H}_{14} \mathrm{~N}_{2} \mathrm{O}_{3}$ & FW & 270.2833 \\
\hline
\end{tabular}
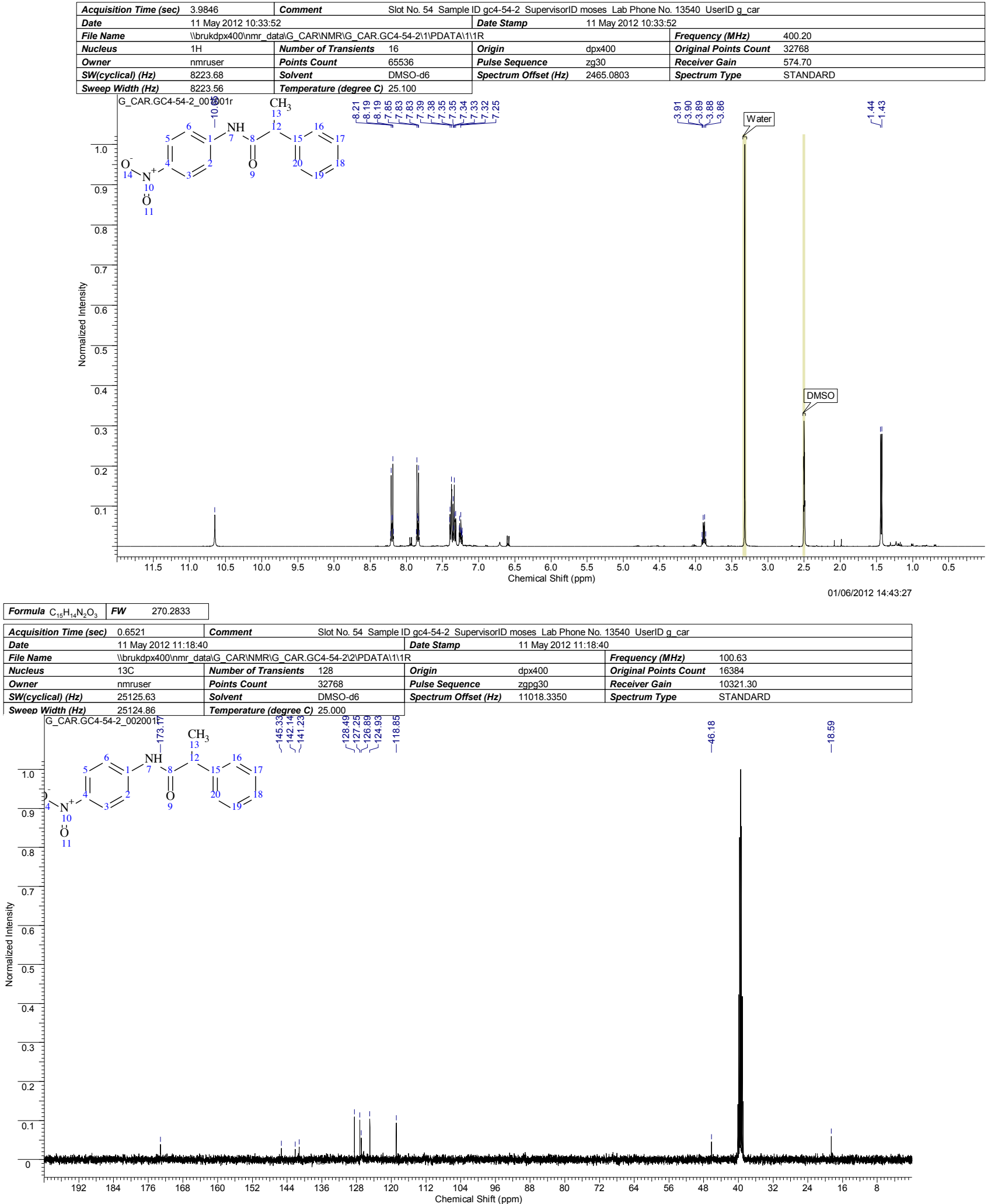

$N$-(4-nitrophenyl)cyclohexanecarboxamide (Table 2, Entry 11) 

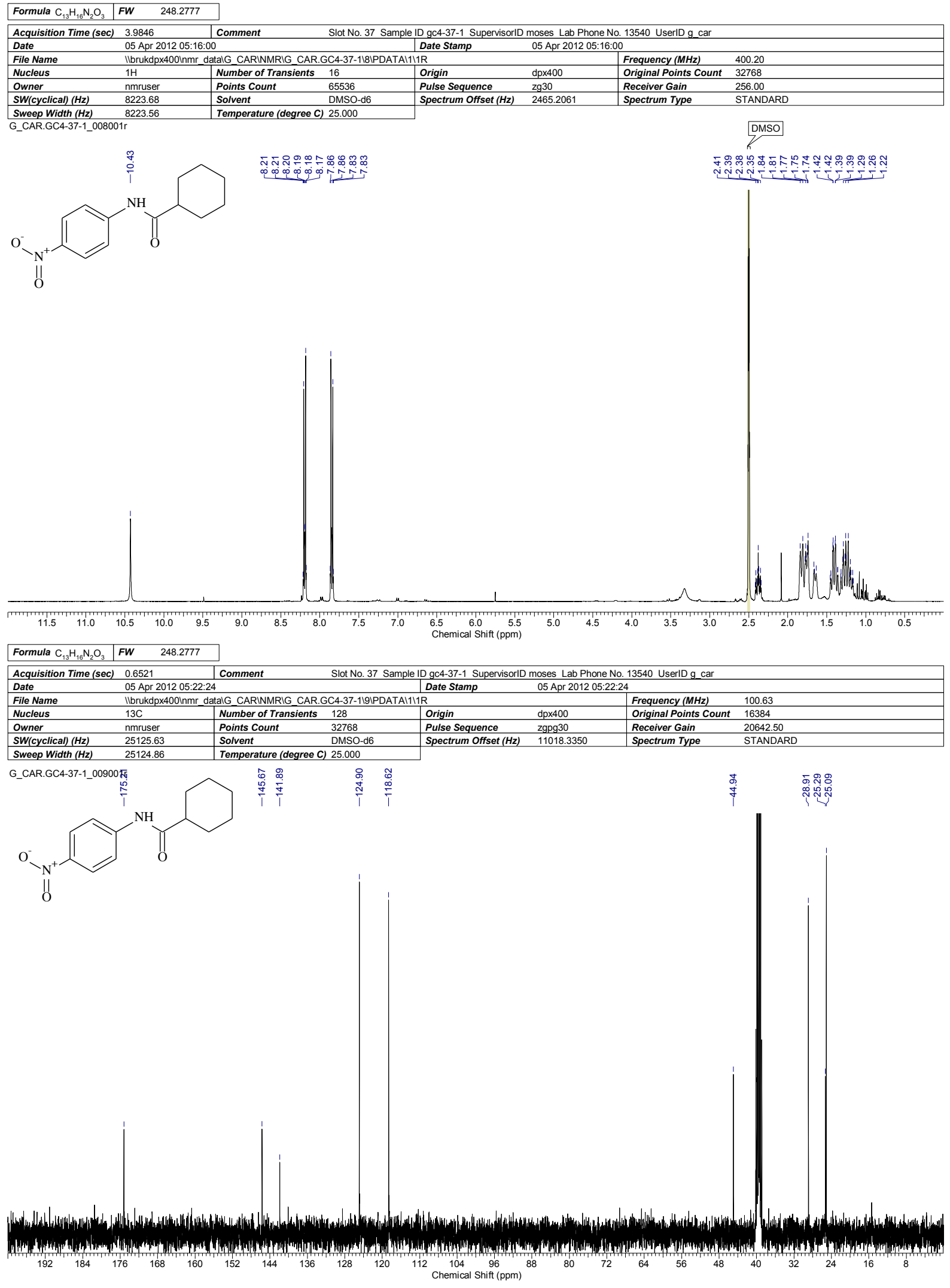

$\mathbf{N}$-(4-nitrophenyl)cyclopropanecarboxamide (Table 2, Entry 12) 

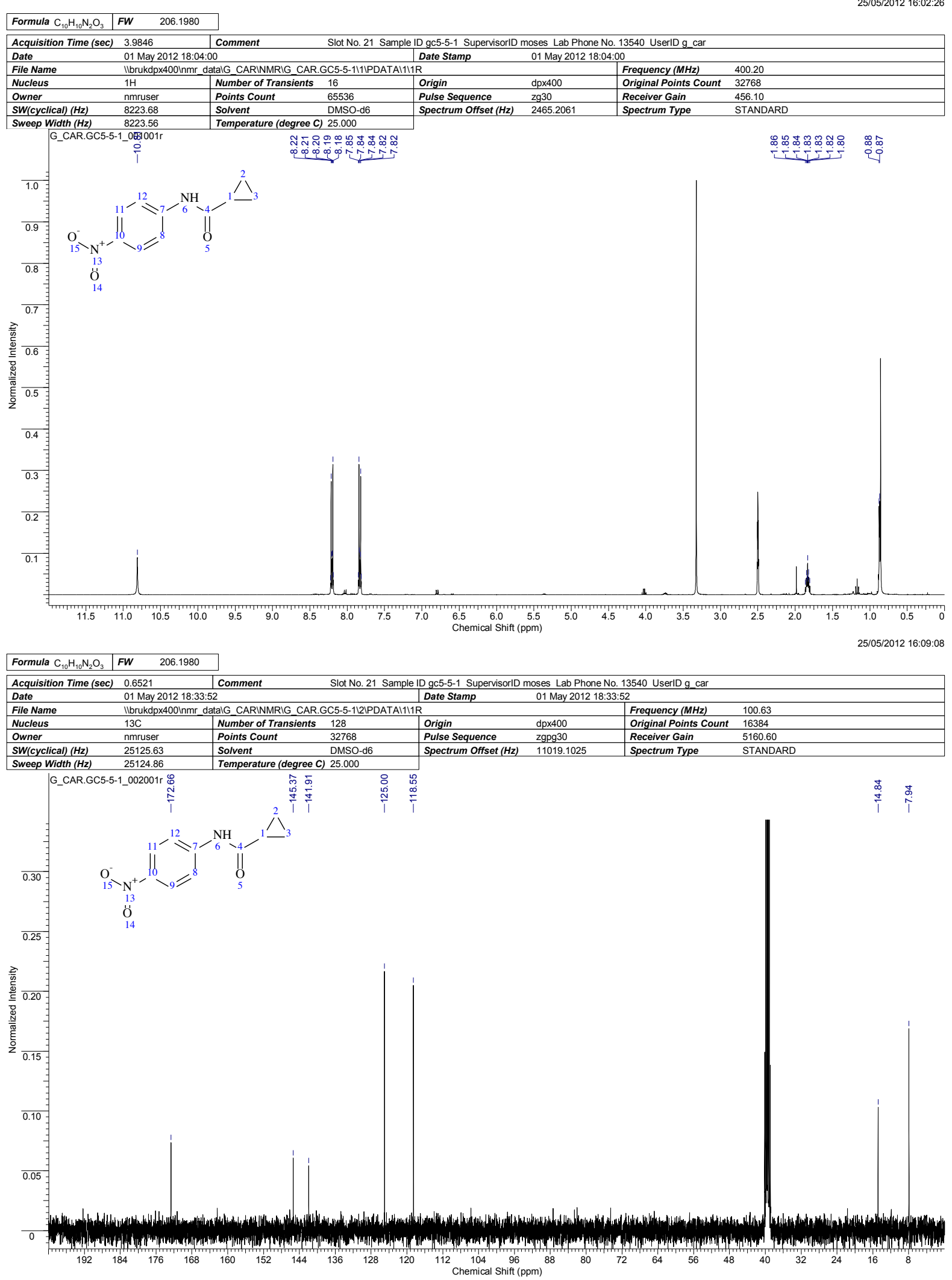

N-(2-chloro-4-nitrophenyl)cyclohexanecarboxamide (Table 2, Entry 13) 

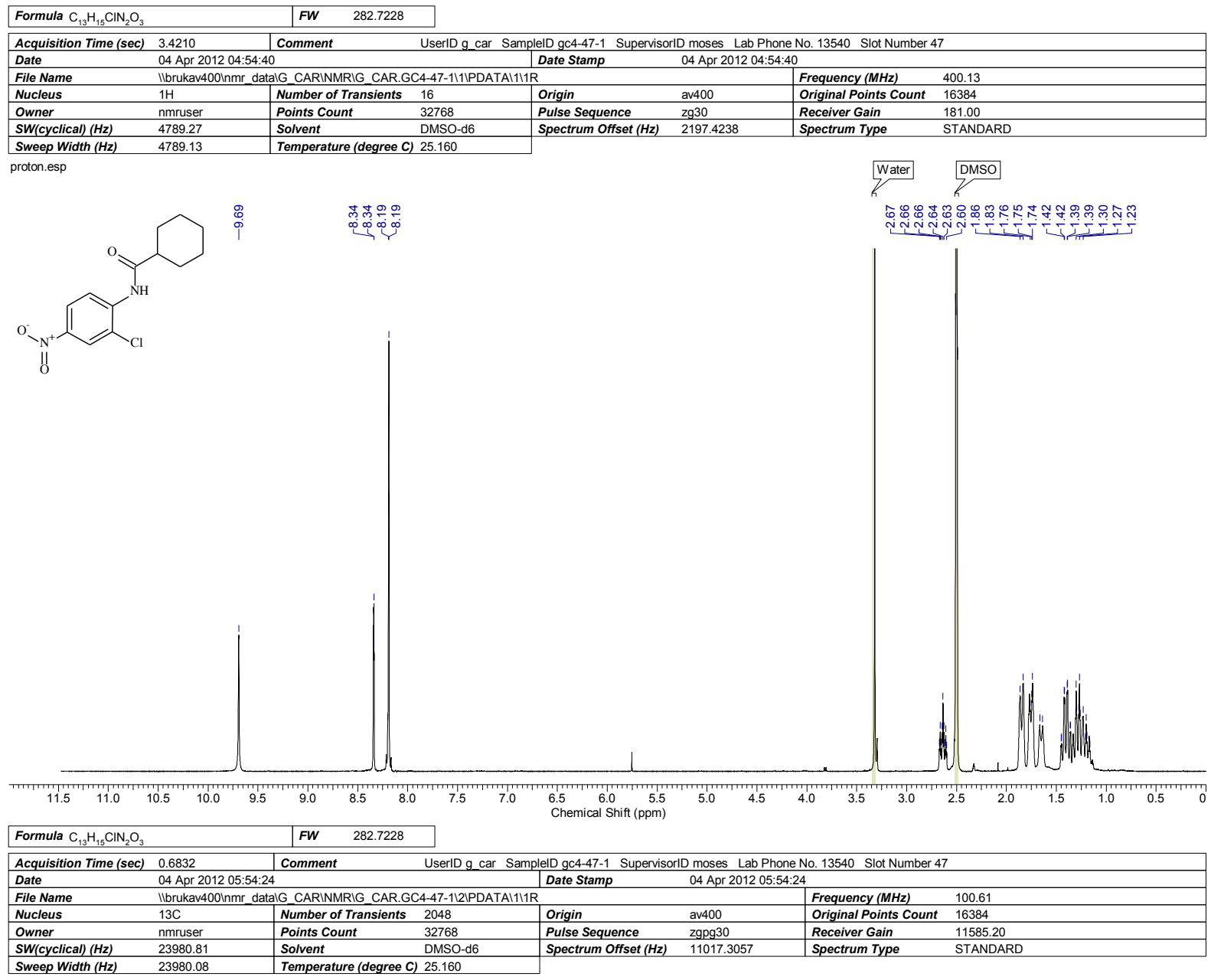
\begin{tabular}{|ll|l|l|ll}
\hline Sweep Width $(\mathrm{Hz})$ & 23980.08 & Temperature (degree C) 25.160 & Spectrum Offset (Hz) 11017.3057 & Spectrum Type & STANDARD \\
\hline
\end{tabular}
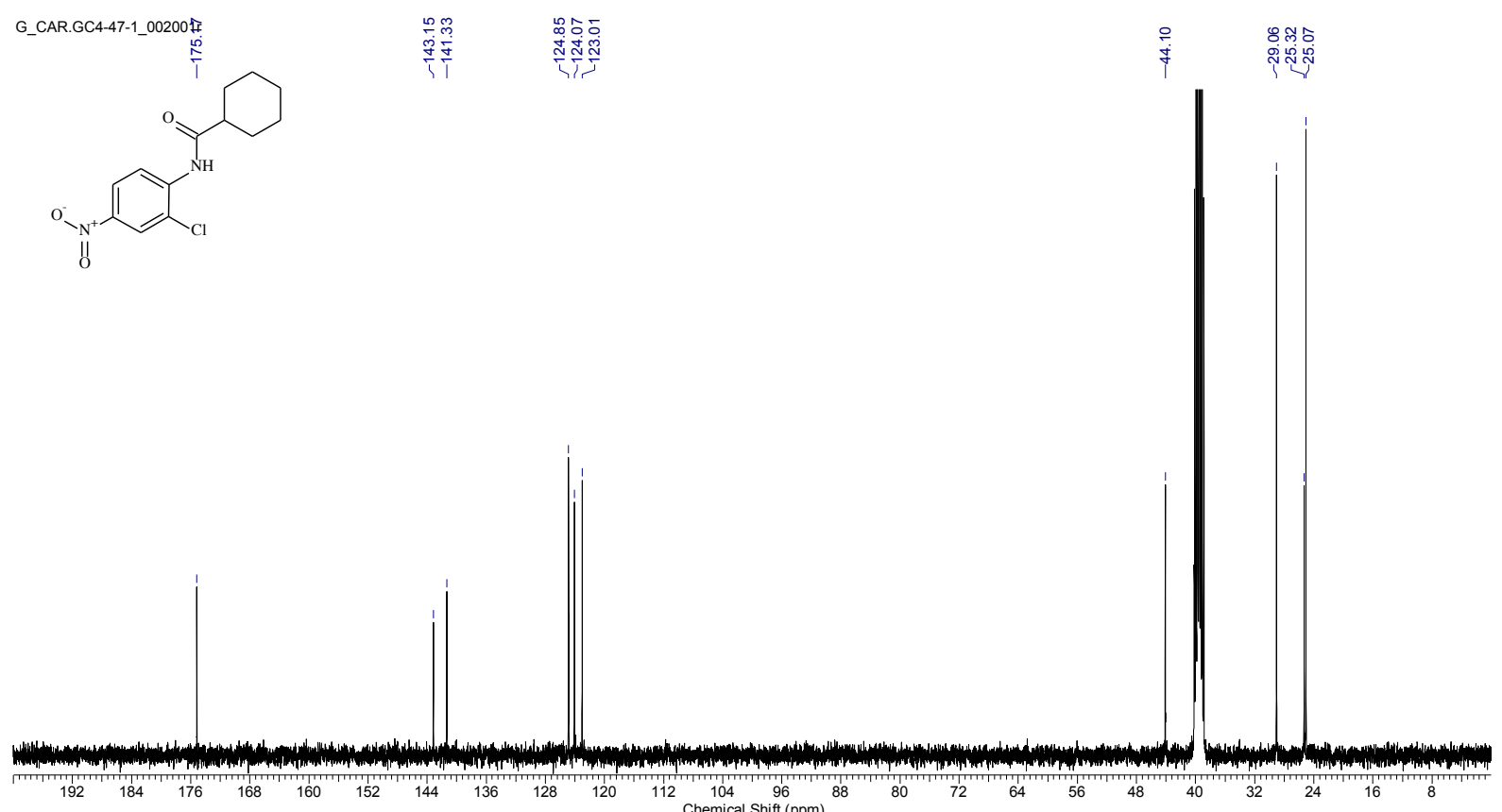

$\boldsymbol{N - ( 4 - n i t r o - 2 - ( t r i f l u o r o m e t h y l ) p h e n y l ) c y c l o h e x a n e c a r b o x a m i d e ~ ( T a b l e ~ 2 , ~ E n t r y ~ 1 4 ) ~}$ 


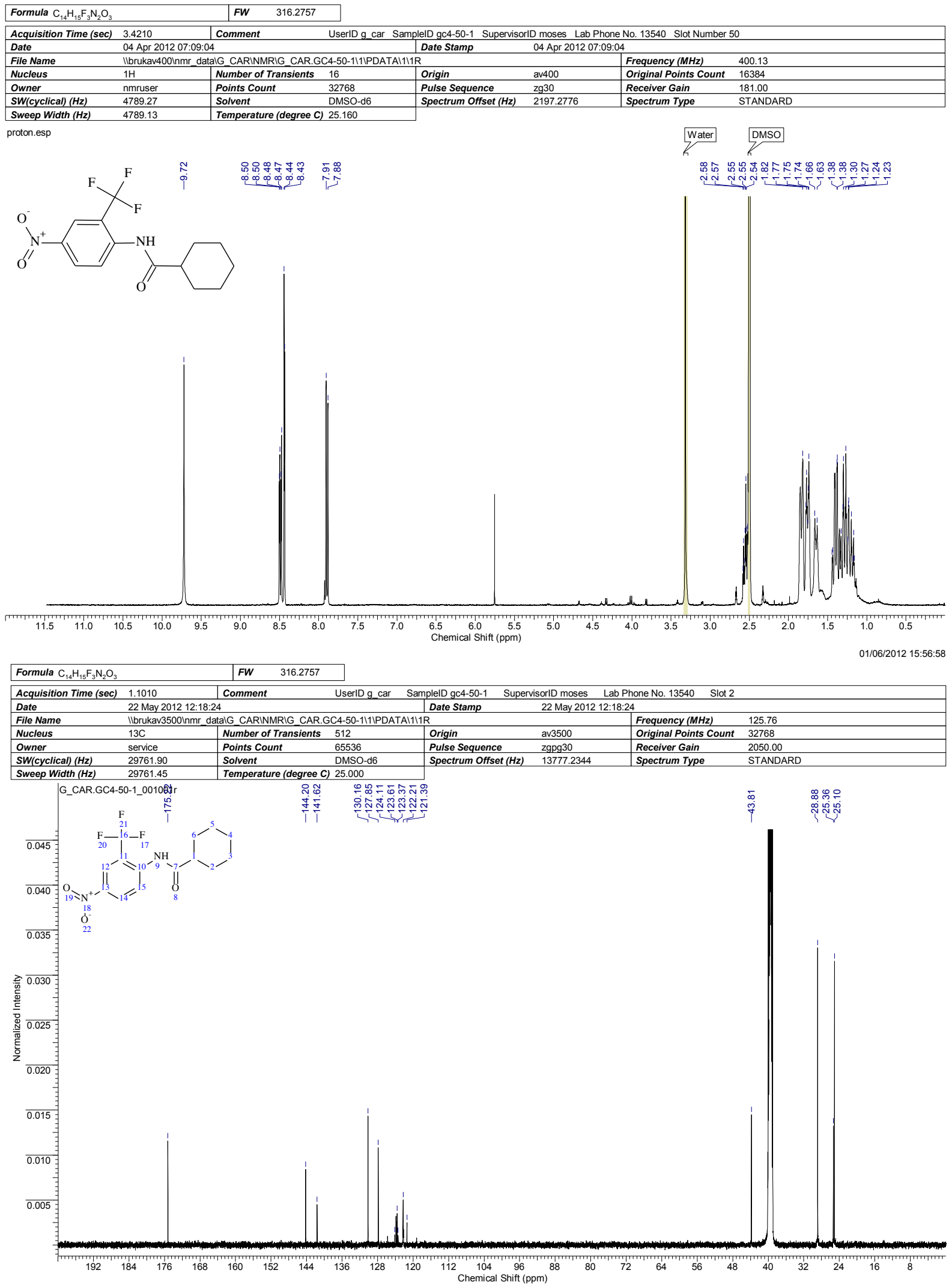

N-(4-nitro-3-(trifluoromethyl)phenyl)cyclohexanecarboxamide (Table 2, Entry 15) 


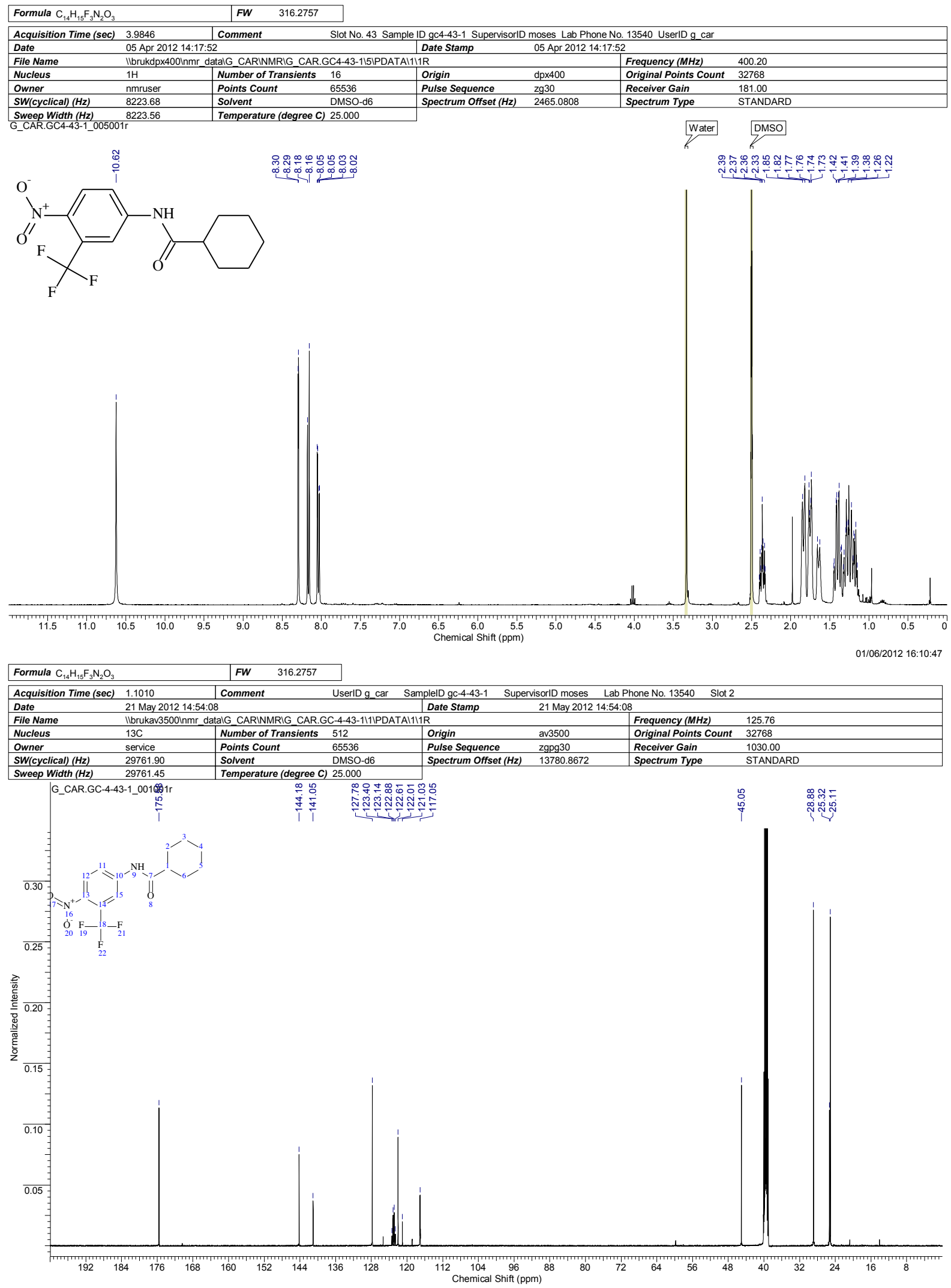


